TRANSACTIONS OF THE

AMERICAN MATHEMATICAL SOCIETY

Volume 363, Number 7, July 2011, Pages 3489-3529

S 0002-9947(2011)05122-6

Article electronically published on February 24, 2011

\title{
NONLINEAR WAVES ON 3D HYPERBOLIC SPACE
}

\author{
JASON METCALFE AND MICHAEL TAYLOR
}

\begin{abstract}
In this article, global-in-time dispersive estimates and Strichartz estimates are explored for the wave equation on three dimensional hyperbolic space. Due to the negative curvature, extra dispersion is noted, as compared to the Euclidean case, and a wider range of Strichartz estimates is proved. Using these, small data global existence to semilinear wave equations is shown for a range of powers that is broader than that known for Euclidean space.
\end{abstract}

\section{INTRODUCTION}

The goal of this article is to study dispersive estimates for the linear wave equation on hyperbolic space and their application to nonlinear wave equations. We will primarily study Strichartz-type estimates. Due to the negative curvature of hyperbolic space, one expects to see more dispersion than in the Euclidean case. This is indeed observed, and a wider range of Strichartz estimates is proved. As such, small data global existence for nonlinear wave equations with power-type nonlinearities can be shown for a larger range of powers.

We shall study the linear wave equation

$$
\begin{gathered}
\left(\partial_{t}^{2}-\Delta\right) u=G(t, x), \\
u(0, \cdot)=f, \quad \partial_{t} u(0, \cdot)=g .
\end{gathered}
$$

A now common measure of dispersion is the class of Strichartz estimates

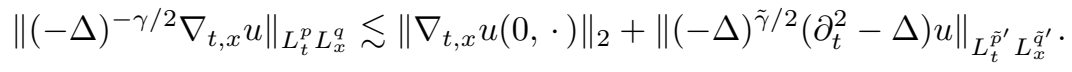

For $(t, x) \in \mathbb{R}_{+} \times \mathbb{R}^{n}$, these estimates originated in the work of Strichartz [25]. It is now known for $(t, x) \in \mathbb{R}_{+} \times \mathbb{R}^{n}$ that (1.2) holds for

$$
\gamma=\frac{n}{2}-\frac{n}{q}-\frac{1}{p}, \quad \frac{2}{p} \leq \frac{n-1}{2}\left(1-\frac{2}{q}\right)
$$

and similarly for $(\tilde{p}, \tilde{q}, \tilde{\gamma})$. The $n=3$ endpoint $(p, q)=(2, \infty)$ is excepted. This is the culmination of progress made by a number of authors. See Keel and Tao [19], where the endpoint estimates were obtained, and the references therein. When $n=3$, this range of indices $(p, q, \gamma)$ is labelled $\mathcal{E}$ in the sequel. See, e.g., Figure 1

When we study (1.1) on $\mathbb{R}_{+} \times \mathcal{H}^{3}$, where $\mathcal{H}^{3}$ is $3 \mathrm{D}$ hyperbolic space with constant sectional curvature -1 , and $\Delta$ represents its Laplace-Beltrami operator, we are able

Received by the editors March 26, 2009.

2000 Mathematics Subject Classification. Primary 35L70.

The second author was partially supported by NSF grants DMS-0800678 and DMS-0758320. 
to obtain a richer family of Strichartz estimates than in the Euclidean setting. In fact, to the set of $(p, q, \gamma)$ satisfying (1.3) can be added the set satisfying

$$
\gamma=1-\frac{2}{q}, \quad \frac{1}{q} \leq \frac{1}{2}-\frac{1}{3 p}
$$

with the $(p, q)=(2,3)$ endpoint case excepted. See Propositions 4.24 .3 This range is labelled $\mathcal{R}$ in Figure 1. The key to proving this extended range of estimates is the dispersive estimate (3.51), given as (1.8) below. Notice that the decay at infinity in this estimate in, say, the $\gamma=1 / 2, q=4$ case, is much better than the $t^{-1 / 2}$ decay which is known in $\mathbb{R}^{1+3}$. In fact, the decay here is comparable to what is obtained in $\mathbb{R}^{1+7}$. The fact that 0 is not in the $L^{p}$-spectrum of $-\Delta$ for $M=\mathcal{H}^{3}$ also has implications for the Strichartz estimates (1.2) in this context.

Closely related estimates of this sort first appeared in Tataru 27. There Strichartz estimates were shown for $\partial_{t}^{2}-\left(\Delta+(n-1)^{2} / 4\right)$. Relations between these estimates and ours are discussed in further detail in Section 3 ,

As an application of such estimates, we consider solutions to semilinear wave equations

$$
\left(\partial_{t}^{2}-\Delta\right) u=F(u)
$$

We seek conditions under which (1.5) can be shown to have a global solution, given small initial data, when $F(u)$ has the form

$$
F(u)=a|u|^{b},
$$

with $b>0, a \in \mathbb{R}$. For $(t, x) \in \mathbb{R}^{1+3}$, it is known that small data global solutions exist for $b>1+\sqrt{2}$. This was first shown by John [17] and is the $n=3$ case of the so-called Strauss conjecture, which is now solved. The Strauss conjecture asserted that, on $\mathbb{R}^{1+n}$, global small data solutions exist for $b>p_{c}$, where $p_{c}$ is the positive root of

$$
(n-1) p^{2}-(n+1) p-2=0 .
$$

See [13 and 27] and the references therein.

On $\mathcal{H}^{3}$, we find that such global existence results hold for a larger range of $b$ than in 3D Euclidean space. Indeed, small data global existence is obtained for $b \geq 5 / 3$. This is obtained merely from Strichartz estimates, and we make no claim of sharpness. In $\mathbb{R}^{3}$, Strichartz estimates only permit one to reach $b \geq$ $3=(n+3) /(n-1)$. The range $1+\sqrt{2}<b<3$ follows from other arguments. Notice here the relationship, which is not coincidental, between the conformal power $(n+3) /(n-1)$ in $n=7$ and the range for which we show global existence.

For some recent related results on dispersive estimates for the Schrödinger equation on hyperbolic space and applications to certain nonlinear equations, the authors refer the interested reader to, e.g., [1, 2, [3, 4, [5, 16. While the techniques involved for the wave equation are related to those employed in the cited works and while the results herein may be heuristically expected based on these previous studies, significant technical obstacles must be overcome to prove the dispersive estimates for the wave equation. In particular, Strichartz estimates typically follow by interpolating an $L^{2}-L^{2}$ energy-type estimate with an $L^{1}-L^{\infty}$ dispersive estimate. For the Schrödinger equation, the latter follows from the form of the representation of the solution. For the wave equation, in Euclidean space, the $L^{1}-L^{\infty}$ estimate requires a more complicated replacement, which typically 
involves Littlewood-Paley theory and Besov spaces. In the absense of an appropriate Littlewood-Paley theory for hyperbolic space, such an estimate is much more elusive in the present setting. To get around this, we make use of Sobolev spaces based on bmo-spaces, and interpolation results from [32, which are reviewed in an appendix to this paper. It is with these tools that the relevant Strichartz estimates are approached.

This paper is organized as follows. In the next section, some explicit formulas are given for the solution operator to (1.1) on $\mathcal{H}^{3}$. Section 3 is dedicated to the main dispersive estimate (3.51), i.e.,

$$
\left\|(-\Delta)^{-s / 2} e^{i t \sqrt{-\Delta}} f\right\|_{L^{q}} \leq \frac{C}{\psi_{\gamma}(t)}\|f\|_{L^{q^{\prime}}}
$$

provided

$$
\gamma \in[0,1), \quad s=2 \gamma, \quad q=\frac{2}{1-\gamma}, \quad q^{\prime}=\frac{2}{1+\gamma},
$$

where

$$
\psi_{\gamma}(t)= \begin{cases}|t|^{\gamma}+|t|^{3 / 2}, & \frac{1}{2} \leq \gamma<1, \\ |t|^{\gamma}+|t|^{3 \gamma}, & 0 \leq \gamma \leq \frac{1}{2} .\end{cases}
$$

Such an estimate follows from the representation of the solution given in Section 2 , together with interpolation arguments, involving bmo-Sobolev spaces. In Section 4. the Strichartz estimates for the homogeneous wave equation, which follow from (1.8), are given. As noted previously, a wider range of exponents is permitted as compared to the wave equation on Euclidean space. Section 5 contains the proofs of Strichartz estimates for nonhomogeneous wave equations. These follow, in what is becoming an increasingly standard argument, from the estimates of Section 4 in concert with the Christ-Kiselev lemma.

Sections 6,8 contain the applications to nonlinear wave equations. The first long-time existence results for nonlinear wave equations are presented in Section 6. These are analogs of known global existence results in Euclidean space for $p \geq(n+3) /(n-1)$, which follow from Strichartz estimates. Due to the richer range of Strichartz estimates, the power in the lower bound for 3-dimensional hyperbolic space resembles that instead of 7-dimensional Euclidean space. In Section 7 the regularity on the initial data which is needed for $b \in[5 / 3,3]$ is improved as opposed to that given in Proposition 6.1. Section 8 is dedicated to proving the existence of global smooth solutions for $\left(\partial_{t}^{2}-\Delta\right) u=a u^{2}$.

The material in Sections [2] is supported by three appendices. Appendex A discusses the telegraph equation and how formulas for its solution yield formulas in Section 2 Appendix B discusses bmo-Sobolev spaces and interpolation results used to prove the dispersive estimates in Section 3. Appendix C gives some general results on the nonbreakdown of smooth solutions to semilinear wave equations, useful in the analysis in Section 8

\section{The SOlution Operator to the WAVE EQUATION ON $\mathbb{R} \times \mathcal{H}^{3}$}

Our goal in this section is to derive an explicit formula for the solution operator

$$
\frac{\sin t \sqrt{-\Delta}}{\sqrt{-\Delta}}
$$


when $\Delta$ is the Laplace-Beltrami operator on $\mathcal{H}^{3}$. We start with the fact that if we set

$$
\begin{aligned}
L & =\Delta+\frac{(n-1)^{2}}{4} \\
& =\Delta+1 \quad(\text { for } n=3),
\end{aligned}
$$

which has the property $\operatorname{Spec}(-L)=[0, \infty)$, then there are exact formulas for various functions of $L$. In particular, for $t>0$,

$$
\frac{\sin t \sqrt{-L}}{\sqrt{-L}} \delta_{y}(x)=\frac{\delta(t-r)}{4 \pi \sinh t},
$$

where $r=r(x, y)$ is the geodesic distance from $x$ to $y$ in $\mathcal{H}^{3}$. A related identity (cf. [30], Chapter 8, (5.15)) is

$$
g(\sqrt{-L}) \delta_{y}(x)=-\frac{1}{(2 \pi)^{3 / 2}} \frac{1}{\sinh r} \frac{\partial}{\partial r} \hat{g}(r),
$$

where again $r=r(x, y)$, and $\hat{g}$ is the Fourier transform of $g$. Note that

$$
\frac{\sin t \sqrt{-\Delta}}{\sqrt{-\Delta}}=S_{t}(\sqrt{-L}), \quad S_{t}(\lambda)=\frac{\sin t \sqrt{\lambda^{2}+1}}{\sqrt{\lambda^{2}+1}} .
$$

One can deduce from the Paley-Wiener theorem that

$$
\text { Supp } \widehat{S}_{t} \subset\{r:|r| \leq|t|\} \text {. }
$$

By comparison, note that

$$
\varphi_{t}(r)=\chi_{[-t, t]}(r) \Longrightarrow \tilde{\varphi}_{t}(\lambda)=2 \frac{\sin t \lambda}{\lambda},
$$

which leads back to (2.3).

Note that $\widehat{S}_{t}(r)$ is the fundamental solution of an equation of Klein-Gordan type on $\mathbb{R} \times \mathbb{R}$. This is not surprising in view of the relation between $L$ and $\Delta$. Here is a general result relating such fundamental solutions.

Proposition 2.1. Let $L_{0}$ be a negative selfadjoint operator and, for a $\in \mathbb{R}$, set

$$
L_{1}=L_{0}+a^{2}, \quad L_{2}=L_{0}-a^{2} .
$$

Then, for $t \in \mathbb{R}$,

$$
\cos t \sqrt{-L_{1}}=\cos t \sqrt{-L_{0}}+a t \int_{0}^{t} \frac{I_{1}\left(a \sqrt{t^{2}-s^{2}}\right)}{\sqrt{t^{2}-s^{2}}} \cos s \sqrt{-L_{0}} d s
$$

and

$$
\cos t \sqrt{-L_{2}}=\cos t \sqrt{-L_{0}}-a t \int_{0}^{t} \frac{J_{1}\left(a \sqrt{t^{2}-s^{2}}\right)}{\sqrt{t^{2}-s^{2}}} \cos s \sqrt{-L_{0}} d s .
$$

Here $J_{1}$ is the Bessel function

$$
J_{1}(\lambda)=\sum_{k=0}^{\infty} \frac{(-1)^{k}}{k !(k+1) !}\left(\frac{\lambda}{2}\right)^{2 k+1}
$$

and

$$
I_{1}(\lambda)=-i J_{1}(i \lambda)
$$


The formulas (2.9) and (2.10) are established in Appendix $\mathrm{A}$, where they are related to formulas for the telegraph equation. By (2.2), we can apply (2.10) with $L_{0}=$ $L, L_{2}=\Delta$ on $\mathcal{H}^{3}$, and $a=1$. Hence, on $\mathcal{H}^{3}$,

$$
\cos t \sqrt{-\Delta}=\cos t \sqrt{-L}-t \int_{0}^{t} \frac{J_{1}\left(\sqrt{t^{2}-s^{2}}\right)}{\sqrt{t^{2}-s^{2}}} \cos s \sqrt{-L} d s .
$$

Note that, for $t>0$,

$$
\cos t \sqrt{-L} \delta_{y}(x)=\frac{\delta^{\prime}(t-r)}{4 \pi \sinh r},
$$

as can be seen by differentiating (2.3) (noting that $\sinh t$ can be replaced by $\sinh r$ in (2.3) $)$. Plugging this into (2.13) yields

$$
\cos t \sqrt{-\Delta} \delta_{y}(x)=\frac{\delta^{\prime}(t-r)}{4 \pi \sinh r}-t \int_{0}^{t} \frac{J_{1}\left(\sqrt{t^{2}-s^{2}}\right)}{\sqrt{t^{2}-s^{2}}} \frac{\partial_{s} \delta(s-r)}{4 \pi \sinh r} d s .
$$

Integrating by parts yields

$$
\frac{\delta^{\prime}(t-r)}{4 \pi \sinh r}-\frac{J_{1}^{\prime}(0) t}{4 \pi \sinh r} \delta(t-r)+\frac{t}{4 \pi \sinh r} \frac{\partial}{\partial r} G\left(\sqrt{t^{2}-r^{2}}\right) \chi_{\{r \leq t\}},
$$

where

$$
G(\lambda)=\frac{J_{1}(\lambda)}{\lambda}
$$

Equivalently,

$$
\begin{aligned}
\cos t \sqrt{-\Delta} \delta_{y}(x)= & \frac{\delta^{\prime}(t-r)}{4 \pi \sinh r}-\frac{J_{1}^{\prime}(0) t}{4 \pi \sinh r} \delta(t-r) \\
& -\frac{t r}{4 \pi \sinh r} \frac{G^{\prime}\left(\sqrt{t^{2}-r^{2}}\right)}{\sqrt{t^{2}-r^{2}}} \chi_{\{r \leq t\}} .
\end{aligned}
$$

Now

$$
\frac{\sin t \sqrt{-\Delta}}{\sqrt{-\Delta}}=\int_{0}^{t} \cos s \sqrt{-\Delta} d s
$$

Applying this to (2.18) gives (for $t>0$ )

$$
\frac{\sin t \sqrt{-\Delta}}{\sqrt{-\Delta}} \delta_{y}(x)=\frac{\delta(t-r)}{4 \pi \sinh r}-\frac{r}{4 \pi \sinh r} \frac{J_{1}\left(\sqrt{t^{2}-r^{2}}\right)}{\sqrt{t^{2}-r^{2}}} \chi_{\{r \leq t\}} .
$$

\section{Dispersive estimates}

Our goal in this section is to use the formula (2.20) to derive dispersive estimates, including the following:

$$
\left\|\frac{\sin t \sqrt{-\Delta}}{\sqrt{-\Delta}} f\right\|_{L^{4}\left(\mathcal{H}^{3}\right)} \leq \frac{C}{\psi(t)}\|f\|_{L^{4 / 3}\left(\mathcal{H}^{3}\right)}, \quad \psi(t)=|t|^{1 / 2}+|t|^{3 / 2},
$$

and others, which will be given further on. The main result will be that (3.51) holds, under the conditions given in 3.45).

To begin, noting that

$$
\frac{\delta(t-r)}{4 \pi \sinh r}=\frac{\delta(t-r)}{4 \pi \sinh t}
$$


and that $J_{1}(\zeta) / \zeta$ is an entire function of $\zeta^{2}$, satisfying

$$
\frac{J_{1}(\zeta)}{\zeta} \sim C \zeta^{-3 / 2} \cos \left(\zeta-\frac{3 \pi}{4}\right)+\cdots, \quad \text { as } \zeta \rightarrow+\infty,
$$

we have the following dispersive estimate. To state it, we take $\lambda>0$ sufficiently large and set

$$
\mathfrak{h}^{s, \infty}\left(\mathcal{H}^{3}\right)=(\lambda I-\Delta)^{-s / 2} \operatorname{bmo}\left(\mathcal{H}^{3}\right) .
$$

See Appendix $B$ for a discussion of these spaces.

Proposition 3.1. Given $f \in L^{1}\left(\mathcal{H}^{3}\right)$,

$$
\left\|\frac{\sin t \sqrt{-\Delta}}{\sqrt{-\Delta}} f\right\|_{\mathfrak{h}^{-1, \infty}} \leq \frac{C}{\varphi(t)}\|f\|_{L^{1}},
$$

where

$$
\varphi(t)=|t|+|t|^{3 / 2} .
$$

Proof. This follows from the estimate

$$
\left\|\frac{\sin t \sqrt{-\Delta}}{\sqrt{-\Delta}} \delta_{y}\right\|_{\mathfrak{h}^{-1, \infty}} \leq \frac{C}{\varphi(t)},
$$

which in turn follows from (2.20). In fact, the second term on the right side of (2.20) has $L^{\infty}$-norm $\leq C / \varphi(t)$, thanks to the estimate

$$
\left|J_{1}(\zeta)\right| \leq C(1+|\zeta|)^{-1 / 2}, \quad \zeta \in[0, \infty) .
$$

As for the first term on the right, it is equal to $\delta(t-r) /(4 \pi \sinh t)$, and we have

$$
\|\delta(t-r)\|_{\mathfrak{h}^{-1, \infty}} \leq C, \quad t \in(0, \infty),
$$

whose proof is essentially the same as that of its well-known Euclidean analogue.

Remark. Note that the estimate (3.5) is stronger as $t \rightarrow+\infty$ than the corresponding estimate with $\mathcal{H}^{3}$ replaced by $\mathbb{R}^{3}$.

We produce further results by interpolating (3.5) with the elementary estimate

$$
\left\|\frac{\sin t \sqrt{-\Delta}}{\sqrt{-\Delta}} f\right\|_{H^{1,2}} \leq C\|f\|_{L^{2}} .
$$

These estimates involve the $L^{p}$-Sobolev spaces

$$
H^{s, p}\left(\mathcal{H}^{3}\right)=(1-\Delta)^{-s / 2} L^{p}\left(\mathcal{H}^{3}\right) .
$$

The relevant interpolation estimates are the following. Assume one has a bounded linear operator

$$
R: L^{2}\left(\mathcal{H}^{3}\right) \rightarrow H^{1,2}\left(\mathcal{H}^{3}\right), \quad R: L^{1}\left(\mathcal{H}^{3}\right) \rightarrow \mathfrak{h}^{-1, \infty}\left(\mathcal{H}^{3}\right),
$$

satisfying

$$
\|R f\|_{H^{1,2}} \leq M_{1}\|f\|_{L^{2}}, \quad\|R f\|_{\mathfrak{h}^{-1, \infty}} \leq M_{0}\|f\|_{L^{1}} .
$$

Then, for $\theta \in(0,1)$,

$$
R: L^{2 /(2-\theta)}\left(\mathcal{H}^{3}\right) \longrightarrow H^{2 \theta-1,2 / \theta}\left(\mathcal{H}^{3}\right),
$$

and (with $C_{\theta}$ independent of $R$ and $f$ )

$$
\|R f\|_{H^{2 \theta-1,2 / \theta}} \leq C_{\theta} M_{1}^{\theta} M_{0}^{1-\theta}\|f\|_{L^{2 /(2-\theta)}} .
$$


This result is an analogue of Corollary 2 in $\S 5$ of [12]; we give further details in Appendix B.

Given (3.15), we deduce that for $\theta \in(0,1)$,

$$
\left\|\frac{\sin t \sqrt{-\Delta}}{\sqrt{-\Delta}} f\right\|_{H^{2 \theta-1,2 / \theta}} \leq \frac{C}{\varphi(t)^{1-\theta}}\|f\|_{L^{2 /(2-\theta)}} .
$$

In particular, taking $\theta=1 / 2$ gives

$$
\left\|\frac{\sin t \sqrt{-\Delta}}{\sqrt{-\Delta}} f\right\|_{L^{4}} \leq \frac{C}{\sqrt{\varphi(t)}}\|f\|_{L^{4 / 3}} .
$$

This is as good as (3.1) for $t \in(0,1]$ but weaker than (3.1) for $t \gg 1$. We need further arguments.

To proceed, we write

$$
\frac{\sin t \sqrt{-\Delta}}{\sqrt{-\Delta}}=R_{0}(t)+R_{1}(t)
$$

with

$$
R_{0}(t)=\frac{\sin t \sqrt{-L}}{\sqrt{-L}}, \quad R_{1}(t) \delta_{y}(x)=-\frac{r}{4 \pi \sinh r} \frac{J_{1}\left(\sqrt{t^{2}-r^{2}}\right)}{\sqrt{t^{2}-r^{2}}} \chi_{\{r \leq t\}} .
$$

Then (3.5) is refined to

$$
\begin{aligned}
& \left\|R_{0}(t) f\right\|_{\mathfrak{h}^{-1, \infty}} \leq \frac{C}{\sinh |t|}\|f\|_{L^{1}}, \\
& \left\|R_{1}(t) f\right\|_{L^{\infty}} \leq \frac{C}{1+|t|^{3 / 2}}\|f\|_{L^{1}} .
\end{aligned}
$$

The estimate (3.10) has the following counterpart:

$$
\begin{aligned}
\left\|\frac{\sin t \sqrt{-L}}{\sqrt{-L}} f\right\|_{H^{1,2}} & \leq C\|\sin t \sqrt{-L} f\|_{L^{2}}+C\left\|\frac{\sin t \sqrt{-L}}{\sqrt{-L}} f\right\|_{L^{2}} \\
& \leq C(1+|t|)\|f\|_{L^{2}},
\end{aligned}
$$

which, in conjunction with (3.10) itself and (3.18)-(3.19), gives

$$
\left\|R_{j}(t) f\right\|_{H^{1,2}} \leq C(1+|t|)\|f\|_{L^{2}}, \quad j=0,1 .
$$

Now an interpolation of (3.20) and (3.23) gives, granted (3.15),

$$
\left\|R_{0}(t) f\right\|_{H^{2 \theta-1,2 / \theta}} \leq C \frac{(1+|t|)^{\theta}}{(\sinh |t|)^{1-\theta}}\|f\|_{L^{2 /(2-\theta)}},
$$

and, in particular,

$$
\left\|R_{0}(t) f\right\|_{L^{4}} \leq C \sqrt{\frac{1+|t|}{\sinh |t|}}\|f\|_{L^{4 / 3}} .
$$

Remark. The estimate (3.24) is equivalent to the estimate (23) of [27]. However, there appears to be a gap in the proof of the result given there. The problem arises 
with the estimate (27), which would imply

$$
\left\|(\lambda I-\Delta)^{-1 / 2} R_{0}(t) f\right\|_{L^{\infty}} \leq \frac{C}{\sinh |t|}\|f\|_{L^{1}},
$$

in place of (3.20). However, such an estimate is not true. (In fact, the last line of p. 802 in 27. has a small error, whose correction manifests a logarithmic singularity.) The argument given here closes that gap.

To obtain further operator estimates on $R_{1}(t)$, we fix $y \in \mathcal{H}^{3}$ and take a closer look at

$$
\Phi(t, x)=\frac{r}{\sinh r} \frac{J_{1}\left(\sqrt{t^{2}-r^{2}}\right)}{\sqrt{t^{2}-r^{2}}} \chi_{\{r \leq t\}} .
$$

Let us write this as

$$
\begin{gathered}
\Phi(t, x)=\Phi_{1}(t, x)+\Phi_{2}(t, x), \quad \Phi_{1}(t, x)=\Phi(t, x) \chi_{\{r \leq t / 2\}}, \\
\Phi_{2}(t, x)=\Phi(t, x) \chi_{\{r \geq t / 2\}} .
\end{gathered}
$$

We have, for $t>0$,

$$
\begin{aligned}
\int_{\mathcal{H}^{3}}\left|\Phi_{1}(t, x)\right|^{p} d V(x) & =4 \pi \int_{0}^{t / 2}\left|\frac{r}{\sinh r} \frac{J_{1}\left(\sqrt{t^{2}-r^{2}}\right)}{\sqrt{t^{2}-r^{2}}}\right|^{p} \sinh ^{2} r d r \\
& \leq C \sup _{\lambda \geq t / 2}\left|\frac{J_{1}(\lambda)}{\lambda}\right|^{p} \int_{0}^{t / 2} \frac{r^{p}}{(\sinh r)^{p-2}} d r \\
& \leq C_{p}(1+|t|)^{-3 p / 2}
\end{aligned}
$$

provided $p>2$, and

$$
\begin{aligned}
\int_{\mathcal{H}^{3}}\left|\Phi_{2}(t, x)\right|^{p} d V(x) & =4 \pi \int_{t / 2}^{t}\left|\frac{r}{\sinh r} \frac{J_{1}\left(\sqrt{t^{2}-r^{2}}\right)}{\sqrt{t^{2}-r^{2}}}\right|^{p} \sinh ^{2} r d r \\
& \leq C \sup _{0<\lambda \leq t}\left|\frac{J_{1}(\lambda)}{\lambda}\right|^{p} \int_{t / 2}^{t} \frac{r^{p}}{(\sinh r)^{p-2}} d r \\
& \leq C_{p}\left(t^{p} \vee t^{2}\right) e^{-(p-2) t}
\end{aligned}
$$

provided $p>2$. Consequently,

$$
p>2 \Longrightarrow\|\Phi(t, \cdot)\|_{L^{p}} \leq C_{p}(1+|t|)^{-3 / 2} .
$$

In light of (3.19), we deduce

$$
\left\|R_{1}(t) f\right\|_{L^{p}} \leq C_{p}(1+|t|)^{-3 / 2}\|f\|_{L^{1}}, \quad \forall p>2 .
$$

Since $R_{1}(t) \delta_{y}(x)$ is symmetric in $x$ and $y$, we deduce by duality that

$$
\left\|R_{1}(t) f\right\|_{L^{\infty}} \leq C_{q}(1+|t|)^{-3 / 2}\|f\|_{L^{q}}, \quad \forall q \in[1,2) .
$$

Interpolation of (3.32) and (3.33) yields

$$
\left\|R_{1}(t) f\right\|_{L^{p}} \leq C_{p q}(1+|t|)^{-3 / 2}\|f\|_{L^{q}}, \quad \forall p>2, q \in[1,2) .
$$


If we apply this with $p=4, q=4 / 3$, and combine this with (3.25), we get

$$
\left\|\frac{\sin t \sqrt{-\Delta}}{\sqrt{-\Delta}} f\right\|_{L^{4}} \leq C\left(\sqrt{\frac{1+|t|}{\sinh |t|}}+\frac{1}{(1+|t|)^{3 / 2}}\right)\|f\|_{L^{4 / 3}},
$$

which is equivalent to (3.1).

We want to push on with further dispersive estimates. First, let us recast estimates leading up to (3.35). For the Laplace-Beltrami operator $\Delta$ on $M=\mathcal{H}^{3}$, these results yield, for

$$
\gamma \in\left[\frac{1}{2}, 1\right), \quad s=2 \gamma, \quad q=\frac{2}{1-\gamma}, \quad q^{\prime}=\frac{2}{1+\gamma},
$$

the estimates

$$
\left\|\frac{\sin t \sqrt{-\Delta}}{\sqrt{-\Delta}} f\right\|_{H^{1-s, q}} \leq \frac{C}{|t|^{\gamma}+|t|^{3 / 2}}\|f\|_{L^{q^{\prime}}} .
$$

In more detail, we have from (3.18)-(3.19),

$$
\frac{\sin t \sqrt{-\Delta}}{\sqrt{-\Delta}} f=R_{0}(t) f+R_{1}(t) f, \quad R_{0}(t) f=\frac{\sin t \sqrt{-L}}{\sqrt{-L}} f,
$$

from (3.24) (with $\gamma=1-\theta$ ),

$$
\left\|R_{0}(t) f\right\|_{H^{1-s, q}} \leq C \frac{(1+|t|)^{1-\gamma}}{(\sinh |t|)^{\gamma}}\|f\|_{L^{q^{\prime}}}
$$

(actually valid for $0<\gamma<1$ ), and from (3.34),

$$
\left\|R_{1}(t) f\right\|_{L^{q}} \leq C_{p q}(1+|t|)^{-3 / 2}\|f\|_{L^{p}}, \quad \forall q>2, p \in[1,2) .
$$

Combining (3.39) and (3.40) gives (3.37).

To proceed, we interpolate the endpoint $(\gamma=1 / 2)$ case

$$
\left\|\frac{\sin t \sqrt{-\Delta}}{\sqrt{-\Delta}} f\right\|_{L^{4}} \leq \frac{C}{|t|^{1 / 2}+|t|^{3 / 2}}\|f\|_{L^{4 / 3}}
$$

of (3.37) with the estimate (3.10):

$$
\left\|\frac{\sin t \sqrt{-\Delta}}{\sqrt{-\Delta}} f\right\|_{H^{1,2}} \leq C\|f\|_{L^{2}},
$$

obtaining for

$$
\gamma \in\left[0, \frac{1}{2}\right], \quad s=2 \gamma, \quad q=\frac{2}{1-\gamma}, \quad q^{\prime}=\frac{2}{1+\gamma},
$$

the estimate

$$
\begin{aligned}
\left\|\frac{\sin t \sqrt{-\Delta}}{\sqrt{-\Delta}} f\right\|_{H^{1-s, q}} & \leq \frac{C}{\left(|t|^{1 / 2}+|t|^{3 / 2}\right)^{2 \gamma}}\|f\|_{L^{q^{\prime}}} \\
& \approx \frac{C}{|t|^{\gamma}+|t|^{3 \gamma}}\|f\|_{L^{q^{\prime}}} .
\end{aligned}
$$

Combining this with (3.37), we get for

$$
\gamma \in[0,1), \quad s=2 \gamma, \quad q=\frac{2}{1-\gamma}, \quad q^{\prime}=\frac{2}{1+\gamma},
$$


the estimate

$$
\left\|\frac{\sin t \sqrt{-\Delta}}{\sqrt{-\Delta}} f\right\|_{H^{1-s, q}} \leq \frac{C}{\psi_{\gamma}(t)}\|f\|_{L^{q^{\prime}}}
$$

where

$$
\psi_{\gamma}(t)= \begin{cases}|t|^{\gamma}+|t|^{3 / 2}, & \frac{1}{2} \leq \gamma<1 \\ |t|^{\gamma}+|t|^{3 \gamma}, & 0 \leq \gamma \leq \frac{1}{2}\end{cases}
$$

Note that $\psi_{\gamma}^{-1} \in L^{1}(\mathbb{R})$ for $\gamma \in(1 / 3,1)$.

As opposed to Euclidean space, one has the result (cf. Appendix B) that for each $m, s \in \mathbb{R}, p \in(1, \infty)$,

$$
(-\Delta)^{m / 2}: H^{s, p}(M) \longrightarrow H^{s-m, p}(M),
$$

whenever $M$ is a symmetric space of noncompact type. In particular, this holds for $M=\mathcal{H}^{3}$, and we can apply this to (3.46) to get

$$
\|\sin t \sqrt{-\Delta} f\|_{H^{-s, q}} \leq \frac{C}{\psi_{\gamma}(t)}\|f\|_{L^{q^{\prime}}},
$$

with $s, q, q^{\prime}$ as in (3.45). Working with the formula (2.18) for $\cos t \sqrt{-\Delta} \delta_{y}(x)$, we also obtain

$$
\|\cos t \sqrt{-\Delta} f\|_{H^{-s, q}} \leq \frac{C}{\psi_{\gamma}(t)}\|f\|_{L^{q^{\prime}}}
$$

and putting together (3.49) and (3.50) gives

$$
\left\|e^{i t \sqrt{-\Delta}} f\right\|_{H^{-s, q}} \leq \frac{C}{\psi_{\gamma}(t)}\|f\|_{L^{q^{\prime}}},
$$

again in the context of (3.45).

\section{Strichartz estimates: Homogeneous Case}

We establish mapping properties of the form

$$
T: H^{\gamma, 2}(M) \longrightarrow L^{p}\left(\mathbb{R}, L^{q}(M)\right), \quad M=\mathcal{H}^{3},
$$

where

$$
T f(t, x)=e^{i t \sqrt{-\Delta}} f(x),
$$

or equivalently

$$
T^{*}: L^{p^{\prime}}\left(\mathbb{R}, L^{q^{\prime}}(M)\right) \longrightarrow H^{-\gamma, 2}(M),
$$

where

$$
T^{*} g(x)=\int_{-\infty}^{\infty} e^{-i t \sqrt{-\Delta}} g(t, x) d t .
$$

The estimates associated with (4.1),

$$
\|T f\|_{L_{t}^{p} L_{x}^{q}} \leq C\|f\|_{H^{\gamma, 2}},
$$

are called Strichartz estimates. 
To see when (4.3) holds, we start by writing

$$
\begin{aligned}
\left(T^{*} g, T^{*} g\right)_{H^{-\gamma, 2}} & =\left((-\Delta)^{-\gamma} T^{*} g, T^{*} g\right)_{L^{2}} \\
& =\iint\left((-\Delta)^{-\gamma} e^{i(s-t) \sqrt{-\Delta}} g(t, \cdot), g(s, \cdot)\right)_{L^{2}} d s d t \\
& \leq C \iint\left\|e^{i(s-t) \sqrt{-\Delta}} g(t, \cdot)\right\|_{H^{-2 \gamma, q}}\|g(s, \cdot)\|_{L^{q^{\prime}}} d s d t,
\end{aligned}
$$

the last inequality by (3.48). Now we apply (3.51) to obtain

$$
\left(T^{*} g, T^{*} g\right)_{H^{-\gamma, 2}} \leq C \iint \frac{1}{\psi_{\gamma}(s-t)}\|g(t, \cdot)\|_{L^{q^{\prime}}}\|g(s, \cdot)\|_{L^{q^{\prime}}} d s d t
$$

valid for

$$
\gamma \in[0,1), \quad q^{\prime}=\frac{2}{1+\gamma}, \quad q=\frac{2}{1-\gamma} \quad\left(\text { hence } \gamma=1-\frac{2}{q}\right),
$$

with $\psi_{\gamma}$ given by (3.47), i.e.,

$$
\psi_{\gamma}(t)= \begin{cases}|t|^{\gamma}+|t|^{3 / 2}, & \frac{1}{2} \leq \gamma<1, \\ |t|^{\gamma}+|t|^{3 \gamma}, & 0 \leq \gamma \leq \frac{1}{2}\end{cases}
$$

To proceed, let us set

$$
\Phi_{\gamma} G(t)=\int_{-\infty}^{\infty} \frac{1}{\psi_{\gamma}(s-t)} G(s) d s
$$

We have:

Proposition 4.1. Let $\gamma, q, q^{\prime}$ be as in (4.8). Then (4.1) and (4.3) hold as long as

$$
\Phi_{\gamma}: L^{p^{\prime}}(\mathbb{R}) \longrightarrow L^{p}(\mathbb{R}) .
$$

For a convolution operator such as (4.10) to have the property (4.11), one certainly needs $p^{\prime} \leq 2 \leq p$. To continue, pick $\varphi \in C_{0}^{\infty}(\mathbb{R})$ such that $\varphi(t)=1$ for $|t| \leq 1,0$ for $|t| \geq 2$, and write

$$
\varphi_{\gamma}^{1}(t)=\frac{\varphi(t)}{\psi_{\gamma}(t)}, \quad \varphi_{\gamma}^{2}(t)=\frac{1-\varphi(t)}{\psi_{\gamma}(t)},
$$

and then, with obvious notation,

$$
\Phi_{\gamma}=\Phi_{\gamma}^{1}+\Phi_{\gamma}^{2} .
$$

We have $\varphi_{\gamma}^{1}$ compactly supported and

$$
\varphi_{\gamma}^{1}(t) \sim C|t|^{-\gamma}, \quad|t| \rightarrow 0,
$$

while $\varphi_{\gamma}^{2}$ is smooth and, as $|t| \rightarrow \infty$,

$$
\varphi_{\gamma}^{2}(t) \sim \begin{cases}C|t|^{-3 / 2}, & \frac{1}{2} \leq \gamma<1, \\ C|t|^{-3 \gamma}, & 0 \leq \gamma \leq \frac{1}{2}\end{cases}
$$

We have

$$
\Phi_{\gamma}^{1}: L^{p^{\prime}}(\mathbb{R}) \longrightarrow L^{p}(\mathbb{R}) \quad \text { provided } 0 \leq \gamma \leq \frac{2}{p}, p>2
$$


the endpoint case $\gamma=2 / p$ following from the Hardy-Littlewood-Sobolev estimate, and the cases $\gamma \in[0,2 / p)$ being more elementary. Also $\Phi_{\gamma}^{1}: L^{2}(\mathbb{R}) \rightarrow L^{2}(\mathbb{R})$ as long as $\gamma \in[0,1)$. We also have

$$
\begin{aligned}
\frac{1}{3}<\gamma<1 & \Longrightarrow \varphi_{\gamma}^{2} \in L^{1}(\mathbb{R}) \cap L^{\infty}(\mathbb{R}) \\
& \Longrightarrow \Phi_{\gamma}^{2}: L^{p^{\prime}}(\mathbb{R}) \rightarrow L^{\tilde{p}}(\mathbb{R}), \forall \tilde{p} \in\left[p^{\prime}, \infty\right] \\
& \Longrightarrow \Phi_{\gamma}^{2}: L^{p^{\prime}}(\mathbb{R}) \rightarrow L^{p}(\mathbb{R}), \forall p^{\prime} \in[1,2] .
\end{aligned}
$$

In addition, given $\gamma \in(0,1 / 2]$,

$$
\Phi_{\gamma}^{2}: L^{p^{\prime}}(\mathbb{R}) \longrightarrow L^{p}(\mathbb{R}) \quad \text { provided } 3 \gamma \geq \frac{2}{p}, p>2,
$$

the endpoint case $3 \gamma=2 / p$ again following from the Hardy-Littlewood-Sobolev estimate, and the case $3 \gamma>2 / p$ is more elementary. For $\gamma=0, p=\infty, p^{\prime}=1$ we also have (4.18).

In summary, we have the following result.

Proposition 4.2. Take $\gamma \in[0,1)$, or equivalently $q \in(2, \infty]$, related by

$$
\gamma=1-\frac{2}{q}
$$

Then (4.1) and (4.3) hold for $M=\mathcal{H}^{3}$ provided either

$$
\frac{2}{3 p} \leq \gamma \leq \frac{2}{p} \text { and } p>2
$$

or

$$
p=2 \text { and } \frac{1}{3}<\gamma<1 .
$$

Remark. The Hardy-Littlewood-Sobolev estimate cited above is the following. Consider

$$
H_{\alpha} f(t)=\int_{-\infty}^{\infty} \frac{f(s)}{|t-s|^{\alpha}} d s
$$

Then

$$
\alpha \in(0,1), 1<q<p<\infty, 1+\frac{1}{p}=\alpha+\frac{1}{q} \Longrightarrow H_{\alpha}: L^{q}(\mathbb{R}) \rightarrow L^{p}(\mathbb{R}) .
$$

Consequently

$$
2<p<\infty, \alpha=\frac{2}{p} \Longrightarrow H_{\alpha}: L^{p^{\prime}}(\mathbb{R}) \rightarrow L^{p}(\mathbb{R}) .
$$

Remark. Another way to describe the triples $(p, q, \gamma)$ satisfying (4.19)-(4.21) is to say that (4.19) holds and either

$$
\frac{1}{2}-\frac{1}{p} \leq \frac{1}{q} \leq \frac{1}{2}-\frac{1}{3 p} \text { and } p>2,
$$

Or

$$
p=2 \text { and } 0<\frac{1}{q}<\frac{1}{3} .
$$

When these conditions hold, we say

$$
(p, q, \gamma) \in \mathcal{R}
$$


We next obtain Strichartz estimates for $(1 / p, 1 / q)$ in a different region of the plane, meeting the previous region on the line segment $1 / p+1 / q=1 / 2, p, q>$ 2. The following estimates are closely similar to Euclidean space analogues in dimension 3. As usual, we take $M=\mathcal{H}^{3}$.

Proposition 4.3. The mapping properties (4.1) and (4.3) hold for

$$
\gamma=\frac{3}{2}-\frac{3}{q}-\frac{1}{p}
$$

provided

$$
\frac{1}{p}+\frac{1}{q} \leq \frac{1}{2}, \quad p, q>2 .
$$

If (4.28) and (4.29) hold, we say

$$
(p, q, \gamma) \in \mathcal{E}
$$

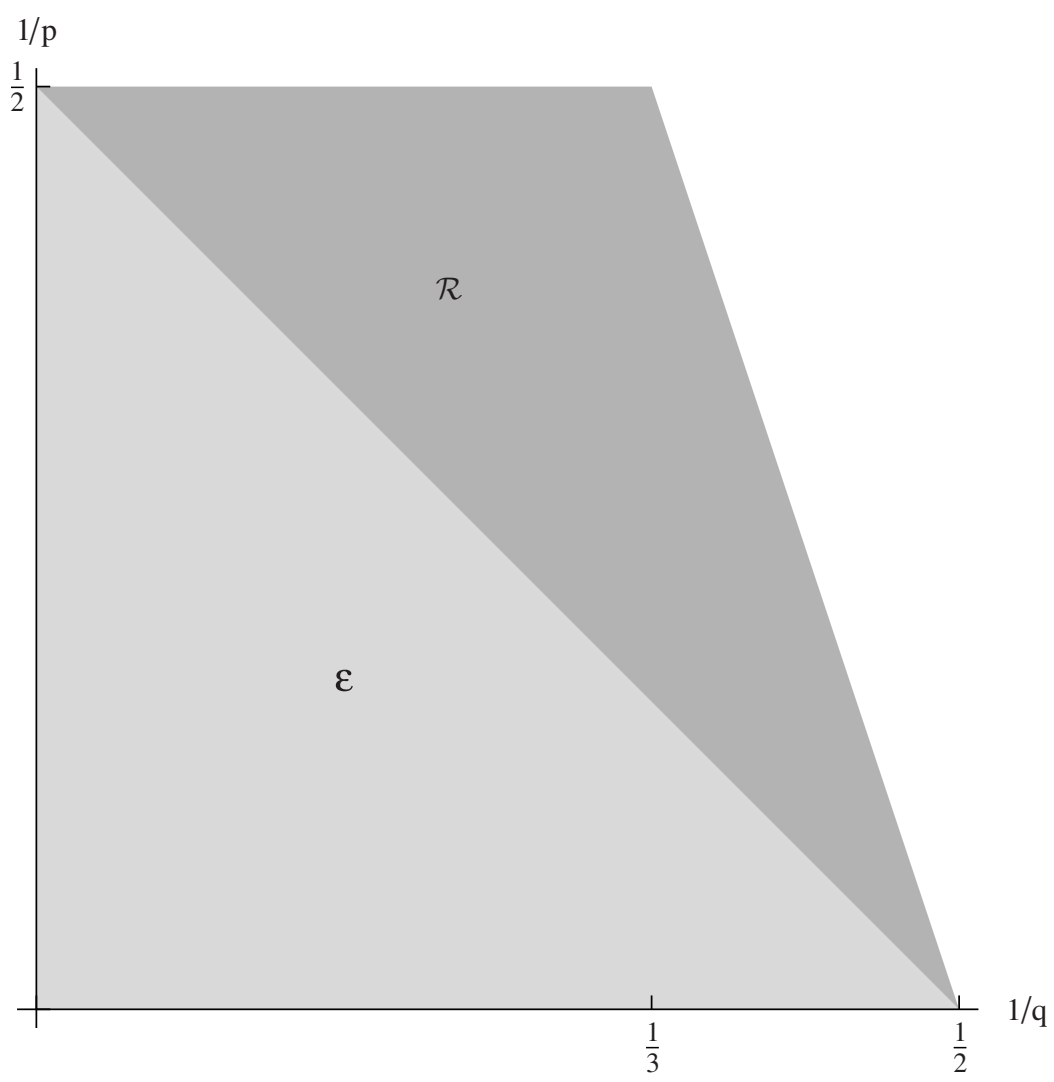

Figure 1. The Strichartz admissible ranges.

Proof. We know from Proposition 4.2 that (4.26) holds for

$$
\gamma=1-\frac{2}{q}
$$


provided (4.20) or (4.21) hold (i.e., $(p, q, \gamma) \in \mathcal{R}$ ), in particular provided

$$
\frac{1}{p}+\frac{1}{q}=\frac{1}{2}, \quad p, q>2 .
$$

Note that if (4.32) holds, then the formulas (4.28) and (4.31) for $\gamma$ coincide. Hence Proposition 4.3 holds when (4.32) holds.

We now deduce Proposition 4.3 more generally. First, whenever (4.28) and (4.32) hold, we can extend (4.26) as follows. We have, for each $\sigma \in \mathbb{R}$,

$$
u=T f \Longrightarrow\|u\|_{L^{p}\left(\mathbb{R}, H^{\sigma, q}(M)\right)} \leq C\|f\|_{H^{\sigma+\gamma}} .
$$

We bring in the Sobolev embedding result:

$$
H^{\sigma, q}(M) \subset L^{q(\sigma)}(M), \quad q(\sigma)=\frac{3 q}{3-\sigma q},
$$

valid for $\sigma q<3$, when $M=\mathcal{H}^{3}$. Thus we obtain the following estimates:

$$
\|u\|_{L^{p}\left(\mathbb{R}, L^{q(\sigma)}(M)\right)} \leq C\|f\|_{H^{\sigma+\gamma}} .
$$

We claim that, for all such $\sigma$ as occur in (4.34), $(p, q(\sigma), \sigma+\gamma) \in \mathcal{E}$, i.e., we have the implication

$$
\begin{aligned}
& \tilde{q}=q(\sigma)=\frac{3 q}{3-\sigma q}, \quad \tilde{\gamma}=\sigma+\gamma \\
& \Longrightarrow \tilde{\gamma}=\frac{3}{2}-\frac{3}{\tilde{q}}-\frac{1}{p} .
\end{aligned}
$$

In fact,

$$
\begin{aligned}
\frac{3}{2}-\frac{3}{\tilde{q}}-\frac{1}{p} & =\frac{3}{2}-\frac{3-\sigma q}{q}-\frac{1}{p} \\
& =\frac{3}{2}-\frac{3}{q}-\frac{1}{p}+\sigma \\
& =\gamma+\sigma,
\end{aligned}
$$

which gives (4.36). We also have the converse result: each $(\tilde{p}, \tilde{q}, \tilde{\gamma}) \in \mathcal{E}$ can be written as $(p, q(\sigma), \sigma+\gamma)$, with $(p, q, \gamma)$ satisfying (4.31) (4.32) and $\sigma \in[0,3 / q)$, so $q(\sigma) \in[q, \infty)$. Thus we have Proposition 4.3.

Let us recall that $T$ is given by

$$
T f(t, x)=e^{i t \sqrt{-\Delta}} f(x) .
$$

It is clear that $T$ commutes with $(\lambda I-\Delta)^{-\gamma / 2}$, so we have the following useful variant of Propositions 4.24 .3 .

Proposition 4.4. Given $(p, q, \gamma) \in \mathcal{R} \cup \mathcal{E}, M=\mathcal{H}^{3}$, we have

$$
T: L^{2}(M) \longrightarrow L^{p}\left(\mathbb{R}, H^{-\gamma, q}(M)\right)
$$

and

$$
T^{*}: L^{p^{\prime}}\left(\mathbb{R}, H^{\gamma, q^{\prime}}(M)\right) \longrightarrow L^{2}(M) .
$$




\section{Strichartz estimates: Inhomogeneous case}

Our goal in this section is to establish the following.

Proposition 5.1. Define $V f=u$ by

$$
\left(\partial_{t}^{2}-\Delta\right) u=f \quad \text { on } \mathbb{R} \times M, \quad u(0)=\partial_{t} u(0)=0 .
$$

As usual, $M=\mathcal{H}^{3}$. Then, whenever

$$
(p, q, \gamma) \text { and }(\tilde{p}, \tilde{q}, \tilde{\gamma}) \in \mathcal{R} \cup \mathcal{E}, \quad \text { and }(p, \tilde{p}) \neq(2,2),
$$

we have

$$
V: L^{\tilde{p}^{\prime}}\left(\mathbb{R}, H^{\tilde{\gamma}, \tilde{q}^{\prime}}(M)\right) \longrightarrow L^{p}\left(\mathbb{R}, H^{1-\gamma, q}(M)\right) .
$$

To begin, we note that the solution to (5.1) is given by

$$
u(t)=\int_{0}^{t} \frac{\sin (t-s) \sqrt{-\Delta}}{\sqrt{-\Delta}} f(s, \cdot) d s .
$$

Bringing in (3.48), we see that to prove Proposition 5.1 it suffices to show that when (5.2) holds,

$$
V_{0}: L^{\tilde{p}^{\prime}}\left(\mathbb{R}, H^{\tilde{\gamma}, \tilde{q}^{\prime}}(M)\right) \longrightarrow L^{p}\left(\mathbb{R}, H^{-\gamma, q}(M)\right),
$$

where

$$
V_{0} f(t, x)=\int_{0}^{t} e^{i(t-s) \sqrt{-\Delta}} f(s, x) d s .
$$

In turn, we can write (5.6), restricted to $t \in \mathbb{R}^{+}$, as

$$
V_{1} g(t, x)=\int_{\infty}^{t} K(t, s) g(s, x) d s,
$$

where

$$
K(t, s)=e^{i(t-s) \sqrt{-\Delta}}
$$

is an operator-valued function of $s$ and $t$ and

$$
g(s, x)=\chi_{\mathbb{R}^{+}}(s) f(s, x) .
$$

There is an analogous formula for (5.6) restricted to $\mathbb{R}^{-}$.

A general result of [8] obtains bounds on (5.7) given bounds on $W$, defined by

$$
W g(t, x)=\int_{-\infty}^{\infty} K(t, s) g(s, x) d s .
$$

If $1 \leq \tilde{p}^{\prime}<p \leq \infty, X$ and $Y$ are Banach spaces of functions, and $g(s, \cdot)$ takes values in $X$, then one has

$$
W: L^{\tilde{p}^{\prime}}(\mathbb{R}, X) \rightarrow L^{p}(\mathbb{R}, Y) \Longrightarrow V_{1}: L^{\tilde{p}^{\prime}}(\mathbb{R}, X) \rightarrow L^{p}(\mathbb{R}, Y) .
$$

Given this, Proposition 5.1 is a consequence of the following.

Lemma 5.2. In the setting of Proposition 5.1, for

$$
W g(t, x)=\int_{-\infty}^{\infty} e^{i(t-s) \sqrt{-\Delta}} g(s, x) d s,
$$

we have

$$
W: L^{\tilde{p}^{\prime}}\left(\mathbb{R}, H^{\tilde{\gamma}, \tilde{q}^{\prime}}(M)\right) \longrightarrow L^{p}\left(\mathbb{R}, H^{-\gamma, q}(M)\right)
$$


Proof. Recalling $T$, given by

$$
T f(t, x)=e^{i t \sqrt{-\Delta}} f(x),
$$

we see that

$$
W=T T^{*} .
$$

Then (5.13) follows from (4.39) and the variant of (4.40):

$$
T^{*}: L^{\tilde{p}^{\prime}}\left(\mathbb{R}, H^{\tilde{\gamma}, \tilde{q}^{\prime}}(M)\right) \longrightarrow L^{2}(M) .
$$

Remark. Regarding the hypothesis (5.2), we can let $(p, \tilde{p})=(2,2)$ in Lemma 5.2 . but to apply [8] and deduce (5.5) we need $\tilde{p}^{\prime}<p$; hence $(p, \tilde{p}) \neq(2,2)$.

Here are some variants of the conclusion of Proposition 5.1

Proposition 5.3. In the setting of Proposition 5.1, in addition to (5.3), i.e.,

$$
\|u\|_{L^{p}\left(\mathbb{R}, H^{1-\gamma, q}(M)\right)} \leq C\|f\|_{L^{\tilde{p}^{\prime}}\left(\mathbb{R}, H^{\tilde{\gamma}}, \tilde{q}^{\prime}(M)\right)},
$$

we also have

$$
\left\|\nabla_{t, x} u\right\|_{L^{p}\left(\mathbb{R}, H^{-\gamma, q}(M)\right)} \leq C\|f\|_{L^{\tilde{p}^{\prime}}\left(\mathbb{R}, H^{\tilde{\gamma}, \tilde{q}^{\prime}}(M)\right)} .
$$

More generally, for each $\sigma \in \mathbb{R}$,

$$
\|u\|_{L^{p}\left(\mathbb{R}, H^{\sigma+1-\gamma, q}(M)\right)} \leq C\|f\|_{L^{\tilde{p}^{\prime}}\left(\mathbb{R}, H^{\sigma+\tilde{\gamma}, \tilde{q}^{\prime}}(M)\right)}
$$

and

$$
\left\|\nabla_{t, x} u\right\|_{L^{p}\left(\mathbb{R}, H^{\sigma-\gamma, q}(M)\right)} \leq C\|f\|_{L^{\tilde{p}^{\prime}}\left(\mathbb{R}, H^{\sigma+\tilde{\gamma}, \tilde{q}^{\prime}}(M)\right)} .
$$

Proof. These are easy consequences of (5.3) and (5.5), the commutativity of $V$ and $(-\Delta)^{-\sigma / 2}$, and the representation

$$
\partial_{t} u(t)=\int_{0}^{t} \cos (t-s) \sqrt{-\Delta} f(s, \cdot) d s .
$$

We are also interested in the behavior of solutions $u(t)$ to (5.1) for fixed $t$, which we denote by

$$
\mathcal{V}^{t} f(x)=V f(t, x)
$$

The behavior is the same as that of

$$
\begin{aligned}
v(t) & =\int_{0}^{t} \frac{e^{i(t-s) \sqrt{-\Delta}}}{\sqrt{-\Delta}} f(s, \cdot) d s \\
& =\frac{e^{i t \sqrt{-\Delta}}}{\sqrt{-\Delta}} T^{*}\left(\chi_{[0, t]} f\right),
\end{aligned}
$$

with $T^{*}$ as in (4.4), the last identity holding at least for $t \geq 0$, with an analogous formula holding for $t \leq 0$. We have

$$
(p, q, \gamma) \in \mathcal{R} \cup \mathcal{E} \Longrightarrow T^{*}: L^{p^{\prime}}\left(\mathbb{R}, L^{q^{\prime}}(M)\right) \rightarrow H^{-\gamma, 2}(M),
$$

and of course $e^{i t \sqrt{-\Delta}} / \sqrt{-\Delta}: H^{-\gamma, 2}(M) \rightarrow H^{1-\gamma, 2}(M)$. We hence have:

Proposition 5.4. In the setting of Proposition 5.1, for each $t \in \mathbb{R}$,

$$
\mathcal{V}^{t}: L^{p^{\prime}}\left(\mathbb{R}, L^{q^{\prime}}(M)\right) \longrightarrow H^{1-\gamma, 2}(M),
$$

with operator norm bound independent of $t$. 
Let us record some special cases of Proposition [5.1] For one, we have

$$
\begin{aligned}
& (p, q, \gamma) \in \mathcal{R} \cup \mathcal{E}, \quad p>2 \\
& \Longrightarrow V: L^{p^{\prime}}\left(\mathbb{R}, H^{\sigma+\gamma, q^{\prime}}(M)\right) \rightarrow L^{p}\left(\mathbb{R}, H^{\sigma+1-\gamma, q}(M)\right), \quad \forall \sigma \\
& \Longrightarrow V: L^{p^{\prime}}\left(\mathbb{R}, L^{q^{\prime}}(M)\right) \rightarrow L^{p}\left(\mathbb{R}, H^{1-2 \gamma, q}(M)\right) .
\end{aligned}
$$

Now if $(p, q, \gamma) \in \mathcal{R}$, so $\gamma=1-2 / q$, then $q \in[2,4] \Rightarrow \gamma \in[0,1 / 2] \Rightarrow 1-2 \gamma \geq 0$, so we have

$$
\begin{aligned}
& (p, q, \gamma) \in \mathcal{R}, \quad 2 \leq q \leq 4 \\
& \Longrightarrow V: L^{p^{\prime}}\left(\mathbb{R}, L^{q^{\prime}}(M)\right) \rightarrow L^{p}\left(\mathbb{R}, L^{q}(M)\right) .
\end{aligned}
$$

Note in particular that

$$
p=q \in\left[\frac{8}{3}, 4\right], \gamma=1-\frac{2}{q} \Longrightarrow(p, q, \gamma) \in \mathcal{R},
$$

from which we deduce:

Proposition 5.5. Take $p \in[8 / 3,4]$. Then, with $M=\mathcal{H}^{3}$,

$$
V: L^{p^{\prime}}(\mathbb{R} \times M) \longrightarrow L^{p}(\mathbb{R} \times M)
$$

and, for each $t \in \mathbb{R}$,

$$
\mathcal{V}^{t}: L^{p^{\prime}}(\mathbb{R} \times M) \longrightarrow H^{2 / p, 2}(M),
$$

with operator norm bound independent of $t$.

\section{Global solutions With Small Data FOR $\square u=a|u|^{b}$}

Here we obtain global solvability results for

$$
\frac{\partial^{2} u}{\partial t^{2}}-\Delta u=F(u), \quad u(0)=\varepsilon f, \quad \partial_{t} u(0)=\varepsilon g,
$$

on $\mathbb{R} \times \mathcal{H}^{3}$, for $\varepsilon$ sufficiently small, provided $f$ and $g$ belong to certain $L^{2}$-Sobolev spaces, in case

$$
F(u)=a|u|^{b} .
$$

Our first result treats small positive $b$. Then we will obtain results valid for larger $b$. The same analysis applies to variants, such as $F(u)=a|u|^{b-1} u$.

Proposition 6.1. Assume $F(u)$ is given by (6.2), with

$$
\frac{5}{3} \leq b \leq 3 \text {. }
$$

There exists $\varepsilon_{0}>0$ with the following property. Take $f \in H^{\gamma, 2}(M), g \in H^{\gamma-1,2}(M)$, $M=\mathcal{H}^{3}$, with

$$
\|f\|_{H^{\gamma, 2}},\|g\|_{H^{\gamma-1,2}} \leq 1, \quad \gamma=\frac{b-1}{b+1} .
$$

Then (6.1) is globally solvable for all $\varepsilon \in\left(0, \varepsilon_{0}\right]$.

Remark. In the next section, we prove Proposition 6.1 with smaller values of $\gamma$ in (6.4). We start here with the less optimal result to minimize the initial technical details. 
To prove Proposition 6.1, we bring in the integral formulation of (6.1):

$$
\begin{aligned}
u(t) & =\varepsilon u_{0}(t)+\int_{0}^{t} \frac{\sin (t-s) \sqrt{-\Delta}}{\sqrt{-\Delta}} F(u(s)) d s \\
& =\varepsilon \Xi^{0}(f, g)(t)+\Xi^{1} u(t) \\
& =\Xi_{\varepsilon} u(t),
\end{aligned}
$$

where

$$
u_{0}(t)=\cos t \sqrt{-\Delta} f+\frac{\sin \sqrt{-\Delta}}{\sqrt{-\Delta}} g .
$$

We take $p \in[8 / 3,4]$ such that

$$
b=\frac{p}{p^{\prime}}=p\left(1-\frac{1}{p}\right)=p-1,
$$

and set (with $M=\mathcal{H}^{3}$ )

$$
\mathfrak{X}_{\delta}^{p}=\left\{v \in L^{p}(\mathbb{R} \times M):\|v\|_{L^{p}(\mathbb{R} \times M)} \leq \delta\right\} .
$$

We will show that there exists $\delta>0$ such that, for all sufficiently small $\varepsilon$,

$$
\Xi_{\varepsilon}: \mathfrak{X}_{\delta}^{p} \longrightarrow \mathfrak{X}_{\delta}^{p} \text { is a contraction map. }
$$

To prove this, first note that, by Proposition 4.2, with $p=q \in[8 / 3,4], \gamma=$ $1-2 / q=1-2 / p$,

$$
\Xi^{0}: H^{1-2 / p, 2}(M) \oplus H^{-2 / p, 2}(M) \longrightarrow L^{p}(\mathbb{R} \times M) .
$$

Note that $\gamma=(b-1) /(b+1)$.

We next need to estimate $\Xi^{1} v$ for $v \in \mathfrak{X}_{\delta}^{p}$. Thus we will need to estimate $\|F(v)\|_{L^{p^{\prime}}(\mathbb{R} \times M)}$. In fact, from (6.2) and (6.7) we have

$$
\begin{aligned}
\|F(v)\|_{L^{p^{\prime}}(\mathbb{R} \times M)} & =|a|\left(\int|v|^{b p^{\prime}} d V\right)^{1 / p^{\prime}} \\
& =|a|\|v\|_{L^{p}(\mathbb{R} \times M)}^{b} .
\end{aligned}
$$

We deduce that

$$
\begin{aligned}
v \in \mathfrak{X}_{\delta}^{p} & \Longrightarrow\|F(v)\|_{L^{p^{\prime}(\mathbb{R} \times M)}} \leq C|a| \delta^{b} \\
& \Longrightarrow\left\|\Xi^{1} v\right\|_{L^{p}(\mathbb{R} \times M)} \leq C|a| \delta^{b},
\end{aligned}
$$

the latter implication via Proposition 5.5. Since $b>1$ when (6.2) holds, we have the mapping property of (6.9); given $\delta>0$ small enough, $\Xi_{\varepsilon}: \mathfrak{X}_{\delta}^{p} \rightarrow \mathfrak{X}_{\delta}^{p}$ for all sufficiently small $\varepsilon$.

It remains to establish the contraction property. To get this, write

$$
\Xi_{\varepsilon} v(t)-\Xi_{\varepsilon} w(t)=\int_{0}^{t} \frac{\sin (t-s) \sqrt{-\Delta}}{\sqrt{-\Delta}} G(v(s), w(s)) d s,
$$

where

$$
\begin{aligned}
G(v(s), w(s)) & =F(v(s))-F(w(s)) \\
& =H(v(s), w(s))(v(s)-w(s)),
\end{aligned}
$$

with

$$
H(v, w)=\int_{0}^{1} F^{\prime}(\tau v+(1-\tau) w) d \tau
$$


Here, $F^{\prime}(v)=a b|v|^{b-1}(\operatorname{sgn} v)$, so

$$
|H(v, w)| \leq C\left(|v|^{b-1}+|w|^{b-1}\right) .
$$

We have

$$
\|G(v, w)\|_{L^{p^{\prime}}(\mathbb{R} \times M)} \leq\|v-w\|_{L^{p}(\mathbb{R} \times M)}\|H(v, w)\|_{L^{r}(\mathbb{R} \times M)},
$$

where $p=b+1$, as in (6.7), and

$$
\begin{aligned}
& \frac{1}{p}+\frac{1}{r}=\frac{1}{p^{\prime}}, \quad \text { hence } r=\frac{p}{p-2}, \\
& \text { hence } r(b-1)=p \text {. }
\end{aligned}
$$

Hence

$$
\begin{aligned}
\|H(v, w)\|_{L^{r}(\mathbb{R} \times M)} & \leq C\left(\left\||v|^{b-1}\right\|_{L^{r}(\mathbb{R} \times M)}+\left\||w|^{b-1}\right\|_{L^{r}(\mathbb{R} \times M)}\right) \\
& \leq C\left(\|v\|_{L^{p}(\mathbb{R} \times M)}^{b-1}+\|w\|_{L^{p}(\mathbb{R} \times M)}^{b-1}\right) .
\end{aligned}
$$

Consequently,

$$
\begin{aligned}
v, w \in \mathfrak{X}_{\delta}^{p} & \Rightarrow\|G(v, w)\|_{L^{p^{\prime}}(\mathbb{R} \times M)} \leq C \delta^{b-1}\|v-w\|_{L^{p}(\mathbb{R} \times M)} \\
& \Rightarrow\left\|\Xi_{\varepsilon} v-\Xi_{\varepsilon} w\right\|_{L^{p}(\mathbb{R} \times M)} \leq C \delta^{b-1}\|v-w\|_{L^{p}(\mathbb{R} \times M)},
\end{aligned}
$$

the latter implication by (6.13) and Proposition 5.5. Since $b>1$, we have the contraction property if $\delta$ is sufficiently small. This completes the proof of Proposition 6.1 .

We next establish the following complement to Proposition 6.1.

Proposition 6.2. The conclusions of Proposition 6.1 also hold for

$$
3<b \leq 5, \quad \gamma=\frac{3}{2}-\frac{2}{b-1} .
$$

In this case we again take $\Xi_{\varepsilon}$ as in (6.5), and we will find $p, q \in[2, \infty)$ and $\delta>0$ such that, with

$$
\mathfrak{X}_{\delta}^{p, q}=\left\{v \in L^{p}\left(\mathbb{R}, L^{q}(M)\right):\|v\|_{L^{p}\left(\mathbb{R}, L^{q}(M)\right)} \leq \delta\right\},
$$

we can show that, for all sufficiently small $\varepsilon$,

$$
\Xi_{\varepsilon}: \mathfrak{X}_{\delta}^{p, q} \longrightarrow \mathfrak{X}_{\delta}^{p, q} \text { is a contraction map. }
$$

Recall that $\Xi_{\varepsilon} u(t)=\varepsilon \Xi^{0}(f, g)(t)+\Xi^{1} u(t)$ and

$$
\Xi^{1} u=V F(u) .
$$

We have

$$
\Xi^{0}: H^{\gamma, 2}(M) \oplus H^{\gamma-1,2}(M) \longrightarrow L^{p}\left(\mathbb{R}, L^{q}(M)\right)
$$

and

$$
V: L^{\tilde{p}^{\prime}}\left(\mathbb{R}, H^{\tilde{\gamma}+\gamma-1, \tilde{q}^{\prime}}(M)\right) \longrightarrow L^{p}\left(\mathbb{R}, L^{q}(M)\right),
$$

for

$$
(p, q, \gamma),(\tilde{p}, \tilde{q}, \tilde{\gamma}) \in \mathcal{E} .
$$

We will impose the condition

$$
\tilde{\gamma}+\gamma=1,
$$


and relate $(\tilde{p}, \tilde{q})$ with $(p, q)$ via the requirement that

$$
F: L^{p}\left(\mathbb{R}, L^{q}(M)\right) \longrightarrow L^{\tilde{p}^{\prime}}\left(\mathbb{R}, L^{\tilde{q}^{\prime}}(M)\right)
$$

when $F$ is given by (6.2). That is to say, we require

$$
p=b \tilde{p}^{\prime}, \quad q=b \tilde{q}^{\prime},
$$

or equivalently

$$
\frac{1}{\tilde{p}}=1-\frac{b}{p}, \quad \frac{1}{\tilde{q}}=1-\frac{b}{q} .
$$

Recalling that if (6.27) holds, then

$$
\gamma=\frac{3}{2}-\frac{3}{q}-\frac{1}{p}, \quad \tilde{\gamma}=\frac{3}{2}-\frac{3}{\tilde{q}}-\frac{1}{\tilde{p}},
$$

we see that (6.28) is equivalent to

$$
\frac{3}{q}+\frac{1}{p}+\frac{3}{\tilde{q}}+\frac{1}{\tilde{p}}=2 .
$$

By (6.31), the left side of (6.33) is

$$
\frac{3}{q}+\frac{1}{p}+3\left(1-\frac{b}{q}\right)+1-\frac{b}{p}=4-(b-1)\left(\frac{3}{q}+\frac{1}{p}\right),
$$

so (6.33) becomes

$$
\frac{1}{p}+\frac{3}{q}=\frac{2}{b-1}
$$

Note that this gives $\gamma=3 / 2-2 /(b-1)$.

For (6.27) to hold we also need $1 / p+1 / q \leq 1 / 2$ and $1 / \tilde{p}+1 / \tilde{q} \leq 1 / 2$. Note that $1 / \tilde{p}+1 / \tilde{q}=2-b(1 / p+1 / q)$, so

$$
\frac{1}{\tilde{p}}+\frac{1}{\tilde{q}} \leq \frac{1}{2} \Longleftrightarrow \frac{1}{p}+\frac{1}{q} \geq \frac{3}{2 b},
$$

which in turn requires $3 / 2 b \leq 1 / 2$, i.e., $b \geq 3$. In the setting of Proposition 6.2 this restriction on $b$ is fine. To satisfy these conditions, we set

$$
\frac{1}{p}+\frac{1}{q}=\frac{3}{2 b}
$$

Now, given $b \geq 3$, we solve (6.35) and (6.37) for $p$ and $q$, obtaining

$$
\frac{1}{p}=\frac{5 b-9}{4 b(b-1)}, \quad \frac{1}{q}=\frac{b+3}{4 b(b-1)} .
$$

Note that

$$
b=3 \Longrightarrow p=q=4, \quad \tilde{p}^{\prime}=\tilde{q}^{\prime}=\frac{4}{3},
$$

agreeing with the treatment for $b=3$ in Proposition 6.1. Some further examples are

$$
\begin{aligned}
& b=4 \Longrightarrow p=\frac{48}{11}, q=\frac{48}{7}, \quad \tilde{p}^{\prime}=\frac{12}{11}, \tilde{q}^{\prime}=\frac{12}{7}, \\
& b=5 \Longrightarrow p=5, q=10, \quad \tilde{p}^{\prime}=1, \quad \tilde{q}^{\prime}=2 .
\end{aligned}
$$

By (6.30), for $\tilde{p}^{\prime}$ and $\tilde{q}^{\prime}$ to be $\geq 1$, we need $p \geq b$ and $q \geq b$. This breaks down for $b>5$, but works for $b \in[3,5]$. 
Now that we have found $p$ and $q$, verifying the existence of $\delta>0$ such that (6.23) holds (for $\varepsilon$ sufficiently small) proceeds as before.

We now complete the circle of global existence results of this section.

Proposition 6.3. The conclusions of Proposition 6.1 also hold for

$$
b \geq 5, \quad \gamma=\frac{3}{2}-\frac{2}{b-1} .
$$

To prove this, we continue to take $\Xi_{\varepsilon}$ as in (6.5), and we will find $\delta>0$ such that, for all sufficiently small $\varepsilon$,

$$
\Xi_{\varepsilon}: \mathfrak{X}_{\delta}^{b} \longrightarrow \mathfrak{X}_{\delta}^{b}
$$

is a contraction map. Here we set

$$
\begin{aligned}
\mathfrak{X}_{\delta}^{b}=\left\{u \in L^{4}\left(\mathbb{R}, H^{\gamma-1 / 2,4}(M)\right) \cap L^{q}(\mathbb{R} \times M):\right. \\
\left.\|u\|_{L^{4}\left(\mathbb{R}, H^{\gamma-1 / 2,4}(M)\right)} \leq \delta,\|u\|_{L^{q}(\mathbb{R} \times M)} \leq \delta\right\},
\end{aligned}
$$

with $\gamma$ as in (6.41) and

$$
q=2(b-1) .
$$

Note that $b \geq 5 \Rightarrow q \geq 8$. (In fact, $b \geq 3 \Rightarrow q \geq 4$.) Also,

$$
\gamma=\frac{3}{2}-\frac{4}{q}, \quad \gamma-\frac{1}{2}=1-\frac{4}{q} .
$$

The distance function we put on $\mathfrak{X}_{\delta}^{b}$ does not involve the norms used in (6.43). Rather it is given as

$$
d(u, v)=\|u-v\|_{L^{4}(\mathbb{R} \times M)} .
$$

The following is a simple but important observation.

Lemma 6.4. $\mathfrak{X}_{\delta}^{b}$, with the metric (6.46), is a complete metric space.

Proof. If $\left(u_{k}\right) \subset \mathfrak{X}_{\delta}^{n}$ is Cauchy in this metric, then there exists $u \in L^{4}(\mathbb{R} \times M)$ such that $u_{k} \rightarrow u$ in norm in $L^{4}(\mathbb{R} \times M)$. The bounds given in (6.43) also imply that a subsequence of $\left(u_{k}\right)$ converges weakly in $L^{4}\left(\mathbb{R}, H^{\gamma-1 / 2,4}(M)\right)$ and in $L^{q}(\mathbb{R} \times M)$ (since these spaces are reflexive) to limits also having these norm bounds. All these limits hold a fortiori in $\mathcal{D}^{\prime}(\mathbb{R} \times M)$, so these limits all coincide. This implies $u \in \mathfrak{X}_{\delta}^{b}$.

Recall that $\Xi_{\varepsilon}(u)=\varepsilon \Xi^{0}(f, g)+V F(u), F(u)=a|u|^{b}$. To show that (6.42) holds, we first note that

$$
\Xi^{0}: H^{\gamma, 2}(M) \oplus H^{\gamma-1,2}(M) \longrightarrow L^{4}\left(\mathbb{R}, H^{\gamma-1 / 2,4}(M)\right),
$$

since $(4,4,1 / 2) \in \mathcal{E}$ and $\Xi^{0}$ commutes with $(1-\Delta)^{\gamma / 2}$. Similarly,

$$
\Xi^{0}: H^{\gamma, 2}(M) \oplus H^{\gamma-1,2}(M) \longrightarrow L^{q}(\mathbb{R} \times M),
$$

for $\gamma$ and $q$ as in (6.41) and (6.44), since also $q \geq 4$ and, in such a case, $(q, q, \gamma) \in \mathcal{E}$. Next, we have, by Proposition 5.3

$$
\|V F(u)\|_{L^{4}\left(\mathbb{R}, H^{\gamma-1 / 2,4}(M)\right)} \leq C\|F(u)\|_{L^{4 / 3}\left(\mathbb{R}, H^{\gamma-1 / 2,4 / 3}(M)\right)},
$$

again because $(4,4,1 / 2) \in \mathcal{E}$. We estimate the right side of (6.49) using the following result. 
Lemma 6.5. For $F(u)=a|u|^{b}, b>1, \sigma=\gamma-1 / 2 \in(0,1)$, and $M=\mathcal{H}^{3}$, or more generally $M$ a Riemannian manifold with $C^{\infty}$ bounded geometry,

$$
\|F(u)\|_{H^{\sigma, 4 / 3}(M)} \leq C\left\|F^{\prime}(u)\right\|_{L^{2}(M)}\|u\|_{H^{\sigma, 4}(M)} .
$$

We will give a proof of this result at the end of this section. First we show how it applies to prove Proposition 6.3. First we have, by (6.50) and Hölder's inequality,

$$
\begin{aligned}
& \|F(u)\|_{L^{4 / 3}\left(\mathbb{R}, H^{\sigma, 4 / 3}(M)\right)} \\
& =\left(\int_{-\infty}^{\infty}\|F(u(s))\|_{H^{\sigma, 4 / 3}(M)}^{4 / 3} d s\right)^{3 / 4} \\
& \leq C\left(\int_{-\infty}^{\infty}\left\|F^{\prime}(u(s))\right\|_{L^{2}(M)}^{2} d s\right)^{1 / 2}\left(\int_{-\infty}^{\infty}\|u(s)\|_{H^{\sigma, 4}(M)}^{4} d s\right)^{1 / 4} \\
& =C\left\|F^{\prime}(u)\right\|_{L^{2}(\mathbb{R} \times M)}\|u\|_{L^{4}\left(\mathbb{R}, H^{\sigma, 4}(M)\right)} .
\end{aligned}
$$

Next

$$
\begin{aligned}
\left|F^{\prime}(u)\right|=a b|u|^{b-1} \Rightarrow\left\|F^{\prime}(u)\right\|_{L^{2}(\mathbb{R} \times M)}^{2} & =C \int_{\mathbb{R}} \int_{M}|u|^{2 b-2} d V d t \\
& =C\|u\|_{L^{q}(\mathbb{R} \times M)}^{q} .
\end{aligned}
$$

Now (6.49)-(6.52) yield

$$
\|V F(u)\|_{L^{4}\left(\mathbb{R}, H^{\gamma-1 / 2,4}(M)\right)} \leq C\|u\|_{L^{q}(\mathbb{R} \times M)}^{q / 2}\|u\|_{L^{4}\left(\mathbb{R}, H^{\gamma-1 / 2,4}(M)\right)} .
$$

It remains to estimate the $L^{q}(\mathbb{R} \times M)$-norm of $V F(u)$. We claim

$$
\|V F(u)\|_{L^{q}(\mathbb{R} \times M)} \leq C\|F(u)\|_{L^{4 / 3}\left(\mathbb{R}, H^{\gamma-1 / 2,4 / 3}(M)\right)},
$$

when $\gamma=3 / 2-4 / q$ and $q \geq 4$. This is equivalent to

$$
\|V g\|_{L^{q}\left(\mathbb{R}, H^{1-\gamma, q}(M)\right)} \leq C\|g\|_{L^{4 / 3}\left(\mathbb{R}, H^{1 / 2,4 / 3}(M)\right)},
$$

which follows from Proposition 5.1. since under our hypotheses,

$$
\left(4,4, \frac{1}{2}\right),(q, q, \gamma) \in \mathcal{E} .
$$

Another application of (6.51)- -6.52$)$ then gives

$$
\|V F(u)\|_{L^{q}(\mathbb{R} \times M)} \leq C\|u\|_{L^{q}(\mathbb{R} \times M)}^{q / 2}\|u\|_{L^{4}\left(\mathbb{R}, H^{\gamma-1 / 2,4}(M)\right)} .
$$

From the estimates (6.47), (6.48), (6.53), and (6.57), it is apparent that one can choose $\delta>0$ such that $\Xi_{\varepsilon}: \mathfrak{X}_{\delta}^{b} \rightarrow \mathfrak{X}_{\delta}^{b}$ for all sufficiently small $\varepsilon$. It remains to guarantee (perhaps after shrinking $\delta$ and $\varepsilon$ further) that this map is a contraction, with respect to the distance function (6.46).

Indeed, for $u, v \in \mathfrak{X}_{\delta}^{b}$,

$$
\begin{aligned}
\left\|\Xi_{\varepsilon}(u)-\Xi_{\varepsilon}(v)\right\|_{L^{4}(\mathbb{R} \times M)} & =\|V(F(u)-F(v))\|_{L^{4}(\mathbb{R} \times M)} \\
& \leq C\|F(u)-F(v)\|_{L^{4 / 3}(\mathbb{R} \times M)},
\end{aligned}
$$

again since $(4,4,1 / 2) \in \mathcal{E}$. Now

$$
\left.|| u\right|^{b}-|v|^{b}|\leq C| u-v \mid\left(|u|^{b-1}+|v|^{b-1}\right),
$$


and hence, since $3 / 4=1 / 2+1 / 4$,

$$
\begin{aligned}
\|F(u)-F(v)\|_{L^{4 / 3}(\mathbb{R} \times M)} & \leq C\left(\left\||u|^{b-1}\right\|_{L^{2}}+\left\||v|^{b-1}\right\|_{L^{2}}\right)\|u-v\|_{L^{4}} \\
& =C\left(\|u\|_{L^{q}}^{b-1}+\|v\|_{L^{q}}^{b-1}\right)\|u-v\|_{L^{4}} .
\end{aligned}
$$

Consequently, for $u, v \in \mathfrak{X}_{\delta}^{b}$,

$$
\left\|\Xi_{\varepsilon}(u)-\Xi_{\varepsilon}(v)\right\|_{L^{4}(\mathbb{R} \times M)} \leq C \delta^{b-1}\|u-v\|_{L^{4}(\mathbb{R} \times M)} .
$$

This proves the contractivity property for $\Xi_{\varepsilon}$ in (6.42). Thus we have Proposition 6.3. modulo the proof of Lemma 6.5. to which we now turn. We formulate a more general result.

Lemma 6.6. Let $M$ be a Riemannian manifold with $C^{\infty}$ bounded geometry. Let $F: \mathbb{R} \rightarrow \mathbb{R}$ be a $C^{1}$ map such that, $F(0)=0$, and, for some $A<\infty$,

$$
|F(u)| \leq C|u| \cdot\left|F^{\prime}(u)\right|
$$

and

$$
\left|F^{\prime}(\tau u+(1-\tau) v)\right| \leq A\left(\left|F^{\prime}(u)\right|+F^{\prime}(v) \mid\right),
$$

for all $u, v \in \mathbb{R}, \tau \in[0,1]$. Then, for $\sigma \in(0,1), p \in(1, \infty)$,

$$
\|F \circ u\|_{H^{\sigma, p}(M)} \leq C\left\|F^{\prime} \circ u\right\|_{L^{q_{1}}(M)}\|u\|_{H^{\sigma, q_{2}}(M)},
$$

provided

$$
\frac{1}{p}=\frac{1}{q_{1}}+\frac{1}{q_{2}}, \quad q_{1} \in(1, \infty], q_{2} \in(1, \infty) .
$$

Note that (6.62) and (6.63) hold for $F(u)=F_{a b}(u)=a|u|^{b}$ as long as $b>1$, the latter since then $\left|F_{a b}^{\prime}(u)\right|$ is monotone in $u$ on $\mathbb{R}^{+}$and on $\mathbb{R}^{-}$. In case $M=\mathbb{R}^{n}$, a result such as Lemma 6.6 (but without (6.63)) was stated in [9], but its proof had a gap, which was filled in 24. In 24 there is a condition more general than (6.63). A still more general result is treated in 31, pp. 112-113. These works establish Lemma 6.6 in case $M=\mathbb{R}^{n}$. We use this to prove Lemma 6.6 for general $M$ with $C^{\infty}$ bounded geometry, in concert with the following result. To state the result, let $\left\{\varphi_{j}: j \in \mathbb{N}\right\}$ be a tame partition of unity, as defined in Appendix B just before Proposition B.2.

Lemma 6.7. In the setting of Lemma 6.6,

$$
\|u\|_{H^{s, p}(M)}^{p} \approx \sum_{j}\left\|\varphi_{j} u\right\|_{H^{s, p}(M)}^{p}+\|u\|_{L^{p}(M)}^{p},
$$

for $s>0, p \in(1, \infty)$.

Meanwhile it is elementary that

$$
\left\|\varphi_{j} u\right\|_{H^{s, p}(M)} \approx\left\|\varphi_{j} u \circ \operatorname{Exp}\right\|_{H^{s, p}\left(\mathbb{R}^{n}\right)},
$$

given $C^{\infty}$ bounded geometry. To see how these results give Lemma 6.6, we have

$$
\begin{aligned}
\|F \circ u\|_{H^{\sigma, p}(M)} & \leq C\left(\sum_{j}\left\|\varphi_{j} F \circ u\right\|_{H^{\sigma, p}}^{p}\right)^{1 / p}+\|F \circ u\|_{L^{p}(M)} \\
& \leq C\left\{\sum_{j}\left(\left\|\varphi_{j} F^{\prime} \circ u\right\|_{L^{q_{1}}}\left\|\varphi_{j} u\right\|_{H^{\sigma, q_{2}}}\right)^{p}\right\}^{1 / p}+\|F \circ u\|_{L^{p}(M)},
\end{aligned}
$$


the second inequality by the case for $\mathbb{R}^{n}$ mentioned above. In turn, this is

$$
\begin{aligned}
& \leq C\left\{\sum_{j}\left\|\varphi_{j} F^{\prime} \circ u\right\|_{L^{q_{1}}}^{q_{1}}\right\}^{1 / q_{1}}\left\{\sum_{j}\left\|\varphi_{j} u\right\|_{H^{\sigma, q_{2}}}^{q_{2}}\right\}^{1 / q_{2}}+\|F \circ u\|_{L^{p}} \\
& \leq C\left\|F^{\prime} \circ u\right\|_{L^{q_{1}}(M)}\|u\|_{H^{\sigma, q_{2}}(M)}+\|F \circ u\|_{L^{p}(M)} .
\end{aligned}
$$

As for the last term, note that (6.62) implies

$$
\begin{aligned}
\|F \circ u\|_{L^{p}(M)} & \leq C\left\||u| \cdot F^{\prime}(u)\right\|_{L^{p}(M)} \\
& \leq C\left\|F^{\prime} \circ u\right\|_{L^{q_{1}}(M)}\|u\|_{L^{q_{2}}(M)} .
\end{aligned}
$$

We conclude this section with a proof of Lemma 6.7 Generalizing (3.11), we have

$$
H^{s, p}(M)=(\lambda I-\Delta)^{-s / 2} L^{p}(M),
$$

for $\lambda \in \mathbb{R}^{+}$sufficiently large; see [32] for more on this. With such a choice of $\lambda$, set

$$
A_{s} u=\sum \psi_{j}(\lambda I-\Delta)^{s / 2} \varphi_{j} u
$$

where $\left\{\psi_{j}: j \in \mathbb{N}\right\}$ is a tame family of elements of $C_{0}^{\infty}(M)$ such that $\psi_{j} \equiv 1$ on $\operatorname{supp} \varphi_{j}$. We have

$$
\begin{gathered}
(\lambda I-\Delta)^{s / 2}=A_{s}+B_{s}, \quad A_{s} \in O P \Psi_{\#}^{s}(M), \\
B_{s} \in O P \Psi_{W}^{-\infty}(M), \quad \forall W<\lambda^{2},
\end{gathered}
$$

where $O P \Psi_{\#}^{s}(M)$ and $O P \Psi_{W}^{-\infty}(M)$ are defined in (B.34)-(B.38). Now we can partition $\mathbb{N}$ into a finite number of sets $S_{\alpha}, 1 \leq \alpha \leq K$, such that for distinct $j, j^{\prime} \in S_{\alpha}, \psi_{j}$ and $\psi_{j^{\prime}}$ have disjoint supports. Write

$$
A_{s}=\sum_{\alpha=1}^{K} A_{s \alpha}, \quad A_{s \alpha}=\sum_{j \in S_{\alpha}} \psi_{j}(\lambda I-\Delta)^{s / 2} \varphi_{j} .
$$

We have

$$
\begin{aligned}
& A_{s \alpha} \in O P \Psi_{\#}^{s}(M), \quad 1 \leq \alpha \leq K, \text { hence } \\
& A_{s \alpha}: H^{s, p}(M) \longrightarrow L^{p}(M) ;
\end{aligned}
$$

cf. 32, Proposition 10.5. Now the support condition gives

$$
\left\|A_{s \alpha} u\right\|_{L^{p}}^{p}=\sum_{j \in S_{\alpha}}\left\|\psi_{j}(\lambda I-\Delta)^{s / 2} \varphi_{j} u\right\|_{L^{p}}^{p},
$$

for each $\alpha \in\{1, \ldots, K\}$; hence

$$
\begin{aligned}
\left\|A_{s} u\right\|_{L^{p}}^{p} & \leq C \sum_{\alpha=1}^{K}\left\|A_{s \alpha} u\right\|_{L^{p}}^{p} \\
& =C \sum_{j}\left\|\psi_{j}(\lambda I-\Delta)^{s / 2} \varphi_{j} u\right\|_{L^{p}}^{p} \\
& \leq C \sum_{j}\left\|\varphi_{j} u\right\|_{H^{s, p}}^{p},
\end{aligned}
$$


so (since $O P \Psi_{W}^{-\infty}(M)$ is bounded on $L^{p}(M)$ for $W$ sufficiently large)

$$
\begin{aligned}
\|u\|_{H^{s, p}}^{p} & \leq C\left\|A_{s} u\right\|_{L^{p}}^{p}+C\left\|B_{s} u\right\|_{L^{p}}^{p} \\
& \leq C \sum_{j}\left\|\varphi_{j} u\right\|_{H^{s, p}}^{p}+C\|u\|_{L^{p}}^{p},
\end{aligned}
$$

which gives half of (6.66).

For the converse, we have

$$
\begin{aligned}
\sum_{j}\left\|\varphi_{j} u\right\|_{H^{s, p}}^{p} & \leq C \sum_{j}\left\|\psi_{j}(\lambda I-\Delta)^{s / 2} \varphi_{j} u\right\|_{L^{p}}^{p}+C \sum_{j}\left\|\varphi_{j} u\right\|_{L^{p}}^{p} \\
& =C \sum_{\alpha=1}^{K} \sum_{j \in S_{\alpha}}\left\{\left\|\psi_{j}(\lambda I-\Delta)^{s / 2} \varphi_{j} u\right\|_{L^{p}}^{p}+\left\|\varphi_{j} u\right\|_{L^{p}}^{p}\right\} \\
& =C \sum_{\alpha=1}^{K}\left\{\left\|\sum_{j \in S_{\alpha}} \psi_{j}(\lambda I-\Delta)^{s / 2} \varphi_{j} u\right\|_{L^{p}}^{p}+\left\|\sum_{j \in S_{\alpha}} \varphi_{j} u\right\|_{L^{p}}^{p}\right\} \\
& \leq C \sum_{\alpha=1}^{K}\left(\left\|A_{s \alpha} u\right\|_{L^{p}}^{p}+\|u\|_{L^{p}}^{p}\right),
\end{aligned}
$$

the third line by the disjoint support condition. By (6.75), this is

$$
\leq C\|u\|_{H^{s, p}(M)} \text {. }
$$

This completes the proof of Lemma 6.7

\section{Improvement of Proposition 6.1}

Here we improve Proposition 6.1, We do not extend the range of $b$ in (6.3), but we lower the needed value of $\gamma$ in (6.4). The argument given here is more closely parallel to that used to prove Proposition 6.2, though with $\mathcal{E}$ replaced by $\mathcal{R}$.

Thus, we take $\Xi_{\varepsilon}$ as in (6.5) and we will find $p, q \in[2, \infty)$ and $\delta>0$ such that, with

$$
\mathfrak{X}_{\delta}^{p, q}=\left\{v \in L^{p}\left(\mathbb{R}, L^{q}(M)\right):\|v\|_{L^{p}\left(\mathbb{R}, L^{q}(M)\right)} \leq \delta\right\},
$$

we can show that, for all sufficiently small $\varepsilon$,

$$
\Xi_{\varepsilon}: \mathfrak{X}_{\delta}^{p, q} \longrightarrow \mathfrak{X}_{\delta}^{p, q} \text { is a contraction map, }
$$

given

$$
\|f\|_{H^{\gamma, 2}},\|g\|_{H^{\gamma-1,2}} \leq 1,
$$

with $\gamma$ somewhat smaller than in (6.4). Recall that $\Xi_{\varepsilon} u(t)=\varepsilon \Xi^{0}(f, g)(t)+\Xi^{1} u(t)$ and

$$
\Xi^{1} u=V F(u)
$$

We have

$$
\Xi^{0}: H^{\gamma, 2}(M) \oplus H^{\gamma-1,2}(M) \longrightarrow L^{p}\left(\mathbb{R}, L^{q}(M)\right)
$$

and

$$
V: L^{\tilde{p}^{\prime}}\left(\mathbb{R}, H^{\tilde{\gamma}+\gamma-1, \tilde{q}^{\prime}}(M)\right) \longrightarrow L^{p}\left(\mathbb{R}, L^{q}(M)\right)
$$

for

$$
(p, q, \gamma),(\tilde{p}, \tilde{q}, \tilde{\gamma}) \in \mathcal{R}
$$


In contrast to (6.28), we impose the condition

$$
\tilde{\gamma}+\gamma \leq 1 \text {. }
$$

As in (6.29), we relate $(\tilde{p}, \tilde{q})$ with $(p, q)$ via the requirement that

$$
F: L^{p}\left(\mathbb{R}, L^{q}(M)\right) \longrightarrow L^{\tilde{p}^{\prime}}\left(\mathbb{R}, L^{\tilde{q}^{\prime}}(M)\right)
$$

when $F(u)=a|u|^{b}$. Thus we require that $p=b \tilde{p}^{\prime}$ and $q=b \tilde{q}^{\prime}$, or equivalently

$$
\frac{1}{\tilde{p}}=1-\frac{b}{p}, \quad \frac{1}{\tilde{q}}=1-\frac{b}{q} .
$$

Recalling that if (7.7) holds, then

$$
\gamma=1-\frac{2}{q}, \quad \tilde{\gamma}=1-\frac{2}{\tilde{q}},
$$

we see that (7.8) is equivalent to

$$
\frac{1}{q}+\frac{1}{\tilde{q}} \geq \frac{1}{2}
$$

Given (7.10), this is equivalent to

$$
\frac{1}{q} \leq \frac{1}{2(b-1)}
$$

as long as $b>1$.

To restate what we have, set

$$
\begin{array}{cc}
x=\frac{1}{p}, y=\frac{1}{q}, & \tilde{x}=\frac{1}{\tilde{p}}, \tilde{y}=\frac{1}{\tilde{q}}, \\
\gamma=1-2 y, & \tilde{\gamma}=1-2 \tilde{y} .
\end{array}
$$

Set

$$
\widetilde{\mathcal{R}}=\left\{(x, y) \in \mathbb{R}^{2}: 0 \leq x \leq \frac{1}{2}, \frac{1}{2}-x \leq y \leq \frac{1}{2}-\frac{x}{3}\right\} \backslash\left\{\left(0, \frac{1}{2}\right),\left(\frac{1}{2}, 0\right),\left(\frac{1}{2}, \frac{1}{3}\right)\right\} .
$$

Then, as long as

$$
(x, y) \in \widetilde{\mathcal{R}}, \quad(\tilde{x}, \tilde{y})=(1-b x, 1-b y) \in \widetilde{\mathcal{R}}, \quad y \leq \frac{1}{2(b-1)},
$$

we have

$$
\begin{aligned}
\Xi^{0}: H^{\gamma, 2}(M) \oplus H^{\gamma-1,2}(M) & \longrightarrow L^{p}\left(\mathbb{R}, L^{q}(M)\right), \\
F: L^{p}\left(\mathbb{R}, L^{q}(M)\right) & \longrightarrow L^{\tilde{p}^{\prime}}\left(\mathbb{R}, L^{\tilde{q}^{\prime}}(M)\right), \text { and } \\
V: L^{\tilde{p}^{\prime}}\left(\mathbb{R}, L^{\tilde{q}^{\prime}}(M)\right) & \longrightarrow L^{p}\left(\mathbb{R}, L^{q}(M)\right) .
\end{aligned}
$$

From (7.17) we deduce, as in the proofs of Propositions 6.1 6.2, the existence of $\varepsilon_{0}, \delta>0$ such that (7.2) holds for all $\varepsilon \in\left(0, \varepsilon_{0}\right]$, given that $f$ and $g$ satisfy (7.3).

A condition equivalent to (7.16) is the following:

$$
\left(x^{\prime}, y^{\prime}\right) \in \widetilde{\mathcal{T}}, \quad(x, y)=\frac{1}{b}\left(x^{\prime}, y^{\prime}\right) \in \widetilde{\mathcal{R}}, \quad y \leq \frac{1}{2(b-1)},
$$

where

$$
\widetilde{\mathcal{T}}=\{(1-\tilde{x}, 1-\tilde{y}):(\tilde{x}, \tilde{y}) \in \widetilde{\mathcal{R}}\}
$$


is the triangle with vertices $(1 / 2,1),(1 / 2,2 / 3)$, and $(1,1 / 2)$, minus these vertices. Here $\left(x^{\prime}, y^{\prime}\right)$ denotes a new set of indices and should not be interpretted as, e.g., Hölder conjugates. Now we observe the following.

For $b=5 / 3$, under the map $(x, y)=b^{-1}\left(x^{\prime}, y^{\prime}\right)$, the vertex $(1 / 2,2 / 3)$ of $\widetilde{\mathcal{T}}$ is taken to $(3 / 10,2 / 5) \in \widetilde{\mathcal{R}}$. Now $(1 / 2,2 / 3) \notin \widetilde{\mathcal{T}}$, but we can deduce that global solvability in Proposition 6.1 holds for

$$
b=\frac{5}{3}, \quad \gamma>\frac{1}{5}
$$

For $5 / 3<b<7 / 3$, the point $(1 / 2, b / 2-1 / 6)$ belongs to the line segment between the vertices $(1 / 2,2 / 3)$ and $(1 / 2,1)$ of $\widetilde{\mathcal{T}}$, and this point is taken by the $\operatorname{map}(x, y)=b^{-1}\left(x^{\prime}, y^{\prime}\right)$ to $(1 / 2 b, 1 / 2-1 / 6 b) \in \widetilde{\mathcal{R}}$. Note that, for $b>1$,

$$
\begin{aligned}
\frac{1}{2}-\frac{1}{6 b} \leq \frac{1}{2(b-1)} & \Longleftrightarrow 3 b^{2}-7 b+1 \leq 0 \\
& \Longleftrightarrow b \leq \frac{7+\sqrt{37}}{6} .
\end{aligned}
$$

Note also that

$$
\begin{gathered}
y=\frac{1}{2}-\frac{1}{6 b} \Longrightarrow 1-2 y=\frac{1}{3 b}, \\
y=\frac{1}{2(b-1)} \Longrightarrow 1-2 y=\frac{b-2}{b-1} .
\end{gathered}
$$

We deduce that global solvability in Proposition 6.1 holds for

$$
\frac{5}{3}<b<\frac{7}{3}, \quad \gamma=\max \left(\frac{1}{3 b}, \frac{b-2}{b-1}\right)= \begin{cases}\frac{1}{3 b}, & \frac{5}{3}<b \leq \frac{7+\sqrt{37}}{6} \\ \frac{b-2}{b-1}, & \frac{7+\sqrt{37}}{6} \leq b<\frac{7}{3}\end{cases}
$$

For $7 / 3 \leq b \leq 3$, the line segment from the vertex $(1 / 2,1)$ of $\tilde{\mathcal{T}}$ to the vertex $(1,1 / 2)$ is taken by the map $(x, y)=b^{-1}\left(x^{\prime}, y^{\prime}\right)$ to a line segment in $\widetilde{\mathcal{R}}$, with endpoints $(1 / 2 b, 1 / b)$ and $(1 / b, 1 / 2 b)$. For such a $b$, we have

$$
\frac{1}{2 b}<\frac{1}{2(b-1)}<\frac{1}{b}
$$

We deduce that global solvability in Proposition 6.1 holds for

$$
\frac{7}{3} \leq b \leq 3, \quad \gamma=\frac{b-2}{b-1}
$$

The result that global solvability in Proposition 6.1 holds for parameters $b$ and $\gamma$ given in (7.20), (7.23), and (7.25) records our improvement of Proposition 6.1

\section{Global Smooth solutions With SMAll DATA FOR $\square u=a u^{2}$}

Here we obtain some global solvability results for

$$
\left(\partial_{t}^{2}-\Delta\right) u=F(u), \quad u(0)=\varepsilon f, \quad \partial_{t} u(0)=\varepsilon g,
$$

on $\mathbb{R} \times \mathcal{H}^{3}$, in case

$$
F(u)=a u^{2},
$$

provided $f, g \in C_{0}^{\infty}\left(\mathcal{H}^{3}\right)$, for $\varepsilon$ sufficiently small. 
To start, we take the integral formulation of (7.1):

$$
\begin{aligned}
u(t) & =\varepsilon u_{0}(t)+\int_{0}^{t} \frac{\sin (t-s) \sqrt{-\Delta}}{\sqrt{-\Delta}} F(u(s)) d s \\
& =\varepsilon \Xi^{0}(f, g)(t)+\Xi^{1} u(t) \\
& =\Xi_{\varepsilon} u(t),
\end{aligned}
$$

where

$$
u_{0}(t)=\cos t \sqrt{-\Delta} f+\frac{\sin t \sqrt{-\Delta}}{\sqrt{-\Delta}} g .
$$

Arguments as in $\sqrt{6}$ show that there exists $\delta>0$ such that if $\mathfrak{X}_{\delta}$ is the complete metric space

$$
\mathfrak{X}_{\delta}=\left\{v \in L^{3}\left(\mathbb{R} \times \mathcal{H}^{3}\right):\|v\|_{L^{3}} \leq \delta\right\}
$$

we have

$$
\Xi_{\varepsilon}: \mathfrak{X}_{\delta} \longrightarrow \mathfrak{X}_{\delta}, \text { a contraction map, }
$$

for sufficiently small $\varepsilon$. This gives global solvability in $\mathfrak{X}_{\delta}$ of $(8.1)-(8.2)$, for $\varepsilon$ sufficiently small.

For such a solution, let us record that

$$
\left\|\varepsilon u_{0}(t)\right\|_{H^{1 / 3,2}(M)} \leq C \varepsilon\left[\|f\|_{H^{1 / 3,2}(M)}+\|g\|_{H^{-2 / 3,2}(M)}\right] .
$$

Also, the second half of Proposition 5.5 gives

$$
\begin{aligned}
\left\|\Xi^{1} u(t)\right\|_{H^{2 / 3,2}(M)} & \leq C\|F(u)\|_{L^{3 / 2}(\mathbb{R} \times M)} \\
& \leq C|a| \delta^{2} .
\end{aligned}
$$

Consequently,

$$
\|u(t)\|_{H^{1 / 3,2}(M)} \leq C \varepsilon\left[\|f\|_{H^{1 / 3,2}(M)}+\|g\|_{H^{-2 / 3,2}(M)}\right]+C|a| \delta^{2} .
$$

We will use this to show that we get global $C^{\infty}$ solutions to (8.1)-8.2), provided that $\varepsilon$ is sufficiently small.

We will make use of some general results on global existence of smooth solutions, presented in Appendix C] To begin, we record the content of Corollary C.2 in this setting.

Proposition 8.1. Let $u$ solve (8.1) (8.2) with $f, g \in C_{0}^{\infty}\left(\mathcal{H}^{3}\right)$. Assume $u$ is a smooth solution for $t \in[0, T)$. Then this solution continues as a smooth solution for $t \in[0, T+\delta)$, for some $\delta>0$, as long as there exist $K_{j}<\infty$ such that

$$
\|u(t)\|_{L^{4}} \leq K_{1}, \quad\|\nabla u(t)\|_{L^{4}} \leq K_{3} .
$$

To proceed, apply Duhamel's formula to write the solution to (8.1)-(8.2) as

$$
u(t)=\varepsilon u_{0}(t)+w(t),
$$

where $u_{0}(t)$ is as in (8.4) and

$$
\begin{aligned}
w(t) & =\int_{0}^{t} \frac{\sin (t-s) \sqrt{-\Delta}}{\sqrt{-\Delta}} F(u(s)) d s \\
& =a \int_{0}^{t} \frac{\sin (t-s) \sqrt{-\Delta}}{\sqrt{-\Delta}} u(s)^{2} d s .
\end{aligned}
$$


By the dispersive estimate (3.1), we have

$$
\begin{aligned}
\|w(t)\|_{L^{4}} & \leq C \int_{0}^{t} \frac{1}{\psi(t-s)}\left\|u(s)^{2}\right\|_{L^{4 / 3}} d s \\
& =C \int_{0}^{t} \frac{1}{\psi(t-s)}\|u(s)\|_{L^{8 / 3}}^{2} d s,
\end{aligned}
$$

with $\psi(t)=|t|^{1 / 2}+|t|^{3 / 2}$. Hence we have

$$
\|u(t)\|_{L^{4}} \leq \varepsilon\left\|u_{0}(t)\right\|_{L^{4}}+C \int_{0}^{t} \frac{1}{\psi(t-s)}\|u(s)\|_{L^{8 / 3}}^{2} d s .
$$

Note also that

$$
\|u\|_{L^{8 / 3}}^{2} \leq C\|u\|_{L^{4}}\|u\|_{L^{2}}
$$

Hence

$$
\|u(t)\|_{L^{4}} \leq \varepsilon\left\|u_{0}(t)\right\|_{L^{4}}+C_{1} \int_{0}^{t} \frac{\|u(s)\|_{L^{2}}\|u(s)\|_{L^{4}}}{\psi(t-s)} d s .
$$

To estimate the second quantity in (8.10), we use the following result.

Lemma 8.2. For $p \in(1, \infty)$ there exists $C_{p} \in(0, \infty)$ such that

$$
C_{p}^{-1}\|\nabla u\|_{L^{p}\left(\mathcal{H}^{3}\right)} \leq\left\|(-\Delta)^{1 / 2} u\right\|_{L^{p}\left(\mathcal{H}^{3}\right)} \leq C_{p}\|\nabla u\|_{L^{p}\left(\mathcal{H}^{3}\right)} .
$$

This is proven in 26, Theorem 6.1. Note that the first inequality in (8.17) follows from (3.48), and in light of (3.48), the second inequality in (8.17) holds if and only if

$$
\|u\|_{L^{p}\left(\mathcal{H}^{3}\right)} \leq C_{p}^{\prime}\|\nabla u\|_{L^{p}\left(\mathcal{H}^{3}\right)} .
$$

Applying $(-\Delta)^{1 / 2}$ to (8.12) and using (8.17), we obtain

$$
\begin{aligned}
\|\nabla w(t)\|_{L^{4}} & \leq C \int_{0}^{t} \frac{1}{\psi(t-s)}\left\|\nabla u(s)^{2}\right\|_{L^{4 / 3}} d s \\
& =2 C \int_{0}^{t} \frac{1}{\psi(t-s)}\|u(s) \nabla u(s)\|_{L^{4 / 3}} d s .
\end{aligned}
$$

Note that

$$
\|f g\|_{L^{4 / 3}} \leq\|f\|_{L^{4}}\|g\|_{L^{2}}
$$

so we obtain

$$
\|\nabla u(t)\|_{L^{4}} \leq \varepsilon\left\|\nabla u_{0}(t)\right\|_{L^{4}}+C_{2} \int_{0}^{t} \frac{\|u(s)\|_{L^{4}}\|\nabla u(s)\|_{L^{2}}}{\psi(t-s)} d s .
$$

It is shown in (C.8) that

$$
\|\nabla u(s)\|_{L^{2}} \leq K_{1} s+E_{1}(0)^{1 / 2},
$$

provided $F(u(t))$ satisfies the conditions given in (C.5), i.e., in this setting,

$$
\|u(t)\|_{L^{4}} \leq \widetilde{K}_{1} .
$$

In such a case, we get from (8.21) that

$$
\|\nabla u(t)\|_{L^{4}} \leq \varepsilon\left\|\nabla u_{0}(t)\right\|_{L^{4}}+C_{2} \int_{0}^{t} \frac{\widetilde{K}_{1}\left(K_{1} s+E_{1}(0)^{1 / 2}\right)}{\psi(t-s)} d s .
$$

Thus we have the following improvement over Proposition 8.1. 
Proposition 8.3. Let $u$ solve (8.1) -(8.2) with $f, g \in C_{0}^{\infty}\left(\mathcal{H}^{3}\right)$. Assume $u$ is a smooth solution for $t \in[0, T)$. Then this solution does not break down as $t \rightarrow T$ as long as there exists $K_{1}<\infty$ such that

$$
\|u(t)\|_{L^{4}} \leq K_{1} .
$$

This in turn holds (by (8.14)) as long as there exists $K_{4}<\infty$ such that

$$
\|u(s)\|_{L^{8 / 3}} \leq K_{4}, \quad \forall s \in[0, T) .
$$

We also have the following.

Proposition 8.4. Let $C_{1}$ be as in (8.16), and take $\varepsilon_{0}>0$ such that

$$
\varepsilon_{0} C_{1} \int_{0}^{\infty} \frac{d t}{\psi(t)} \leq \frac{1}{2}
$$

If $u$ is a smooth solution to (8.1)-(8.2) for $t \in[0, T)$, with $f, g \in C_{0}^{\infty}\left(\mathcal{H}^{3}\right)$, and if

$$
\|u(s)\|_{L^{2}} \leq \varepsilon_{0}, \quad \forall s \in[0, T),
$$

then $u$ continues to a smooth solution of (8.1) on a neighborhood of $[0, T]$.

Proof. From (8.16) we get

$$
\|u(t)\|_{L^{4}} \leq \varepsilon\left\|u_{0}(t)\right\|_{L^{4}}+\frac{1}{2} \sup _{0 \leq s \leq t}\|u(s)\|_{L^{4}},
$$

which yields

$$
\sup _{0 \leq s \leq t}\|u(s)\|_{L^{4}} \leq 2 \varepsilon \sup _{0 \leq s \leq t}\left\|u_{0}(s)\right\|_{L^{4}}
$$

Recalling (8.9), and noting that $\delta$ could be taken to be arbitrarily small (generally forcing $\varepsilon$ to be even smaller), we have the following.

Proposition 8.5. Given $f, g \in C_{0}^{\infty}\left(\mathcal{H}^{3}\right)$, and $F(u)$ as in (8.2), there exists $\varepsilon_{1}>0$ such that (8.1) has a unique solution

$$
u \in C^{\infty}\left(\mathbb{R} \times \mathcal{H}^{3}\right),
$$

as long as $|\varepsilon| \leq \varepsilon_{1}$.

\section{Appendix A. The telegraph equation and Proposition 2.1}

As mentioned in $\$ 2$, the derivation of the formulas (2.9) and (2.10) in Proposition 2.1 is closely related to the analysis of the telegraph equation. We expand on this here and prove those formulas.

To begin, the telegraph equation is the case $n=1$ of

$$
\partial_{t}^{2} u+2 a \partial_{t} u-\Delta u=0, \quad u(0)=f, \quad \partial_{t} u(0)=0,
$$

where $\Delta$ is the Laplace operator on $\mathbb{R}^{n}$. It is well known that if $a>0$, then

$$
n=1, f \geq 0 \Longrightarrow u(t) \geq 0 \text { for } t \geq 0 .
$$

In fact there is a probabilistic formula for $u(t, x)$ in terms of the Poisson process on $\mathbb{R}$; see [6], pp. 280-282. The result (A.2) can be observed from explicit formulas, which we will derive. 
We make some parenthetical comments on solutions to A.11). First, granted sufficient decay of $f$, if $I_{t}(f)=\int_{\mathbb{R}^{n}} u(t, x) d x$, we readily see that

$$
\partial_{t}^{2} I_{t}(f)+2 a \partial_{t} I_{t}(f)=0,\left.\quad \partial_{t} I_{t}(f)\right|_{t=0}=0,
$$

and hence $I_{t}(f) \equiv I_{0}(f)=\int f d x$.

We now relate (A.1) to something closer to the subject matter of Proposition 2.1. Namely, if $u$ solves (A.1), then $v(t, x)=e^{a t} u(t, x)$ solves

$$
\partial_{t}^{2} v-\left(\Delta+a^{2}\right) v=0, \quad v(0)=f, \quad \partial_{t} v(0)=a f .
$$

Hence

$$
e^{a t} u(t)=(\cos t \sqrt{-L}) f+a \frac{\sin t \sqrt{-L}}{\sqrt{-L}} f,
$$

where

$$
L=\Delta+a^{2}
$$

Note that

$$
\int_{0}^{t} \cos s \sqrt{-L} d s=\frac{\sin t \sqrt{-L}}{\sqrt{-L}},
$$

so (A.2) follows from the assertion that as long as $a^{2} \geq 0$ in (A.6),

$$
n=1, f \geq 0 \Longrightarrow(\cos t \sqrt{-L}) f \geq 0 .
$$

We derive a formula for $(\cos t \sqrt{-L}) f=w(t, x)$, solving

$$
\partial_{t}^{2} w-\left(\Delta+a^{2}\right) w=0, \quad w(0)=f, \partial_{t} w(0)=0,
$$

by comparing $w(t)$ with $w_{0}(t)$, solving

$$
\partial_{t}^{2} w_{0}-\Delta w_{0}=0, \quad w_{0}(0)=f, \partial_{t} w_{0}(0)=0 .
$$

Our approach will borrow a trick from Appendix B (pp. 667-668) of [20]. It starts with the identity

$$
e^{t L} f=\frac{1}{2 \pi} \int_{-\infty}^{\infty} \hat{h}_{t}(s) \cos s \sqrt{-L} f d s
$$

with

$$
\hat{h}_{t}(s)=\frac{2 \pi}{\sqrt{4 \pi t}} e^{-s^{2} / 4 t} .
$$

If we set $4 t=1 / \lambda$, we see that $w(t)=(\cos t \sqrt{-L}) f$ satisfies

$$
\int_{0}^{\infty} w(s, x) e^{-\lambda s^{2}} d s=\frac{1}{2} \sqrt{\frac{\pi}{\lambda}} e^{a^{2} / 4 \lambda} e^{(1 / 4 \lambda) \Delta} f(x) .
$$

By comparison, $w_{0}(t)=(\cos t \sqrt{-\Delta}) f$ satisfies

$$
\int_{0}^{\infty} w_{0}(s, x) e^{-\lambda s^{2}} d s=\frac{1}{2} \sqrt{\frac{\pi}{\lambda}} e^{(1 / 4 \lambda) \Delta} f(x) .
$$

In other words,

$$
\int_{0}^{\infty} w(s, x) e^{-\lambda s^{2}} d s=e^{a^{2} / 4 \lambda} \int_{0}^{\infty} w_{0}(s, x) e^{-\lambda s^{2}} d s .
$$


Note that the change of variable $\sigma=s^{2}$ makes A.15) a relation between Laplace transforms of

$$
\psi(\sigma, x)=\frac{w(\sqrt{\sigma}, x)}{\sqrt{\sigma}} \text { and } \psi_{0}(\sigma, x)=\frac{w_{0}(\sqrt{\sigma}, x)}{\sqrt{\sigma}} .
$$

Hence a representation of $e^{a^{2} / 4 \lambda}$ as a Laplace transform:

$$
e^{a^{2} / 4 \lambda}=\int_{0}^{\infty} \varphi(\sigma) e^{-\lambda \sigma} d \sigma
$$

will give rise to a convolution formula:

$$
\psi(\sigma, x)=\int_{0}^{\sigma} \varphi(\tau) \psi_{0}(\sigma-\tau, x) d \tau .
$$

To identify the function $\varphi(\sigma)$ in (A.17), we start with the following:

$$
\int_{0}^{\infty} J_{\nu}(a s) s^{\nu+1} e^{-\lambda s^{2}} d s=\frac{a^{\nu}}{(2 \lambda)^{\nu+1}} e^{-a^{2} / 4 \lambda} .
$$

This is one of the most fundamental Hankel transforms and is used to prove the Hankel inversion formula (cf. Proposition 8.1 in Chapter 8 of 30, especially (8.21); see also (8.32)). We recall that the method of proof was to replace $J_{\nu}(a s)$ in (A.19) by its power series expansion:

$$
J_{\nu}(a s)=\sum_{k=0}^{\infty} \frac{(-1)^{k}}{k ! \Gamma(k+\nu+1)}\left(\frac{a s}{2}\right)^{\nu+2 k},
$$

and integrate term by term. This works for $\nu>-1$. We want to pass to the limit $\nu \searrow-1$. Of course the integrand converges pointwise to $J_{-1}(a s) e^{-\lambda s^{2}}=$ $-J_{1}(a s) e^{-\lambda s^{2}}$. The integral involving each term in (A.20) converges to the integral of the corresponding term in the power series expansion of $J_{-1}(a s)$ (times $e^{-\lambda s^{2}}$ ) except for the term $k=0$. In this case, the integrand converges to 0 pointwise, but the integral does not converge to 0 . Rather, due to the fact that, with $\nu=$ $-1+\varepsilon, B>0$,

$$
\frac{1}{\Gamma(\varepsilon)} \int_{0}^{B} s^{\varepsilon-1} d s=\frac{1}{\varepsilon \Gamma(\varepsilon)} B^{\varepsilon} \rightarrow 1 \text { as } \varepsilon \searrow 0
$$

the result we arrive at is

$$
\int_{0}^{\infty} J_{1}(a s) e^{-\lambda s^{2}} d s=\frac{1-e^{-a^{2} / 4 \lambda}}{a} .
$$

Next we can analytically continue in $a$. We have (cf. (6.55) of [30], Chapter 3)

$$
I_{\nu}(r)=e^{-\pi i \nu / 2} J_{\nu}(i r), \quad r>0,
$$

and, in particular,

$$
I_{1}(r)=-i J_{1}(i r)
$$

Hence, for $a, \lambda>0$,

$$
\int_{0}^{\infty} I_{1}(a s) e^{-\lambda s^{2}} d s=\frac{e^{a^{2} / 4 \lambda}-1}{a}
$$


We note that the power series for $J_{\nu}(z)$ yields

$$
I_{1}(r)=\sum_{k=0}^{\infty} \frac{1}{k !(k+1) !}\left(\frac{r}{2}\right)^{2 k+1} .
$$

In particular,

$$
r>0 \Longrightarrow I_{1}(r)>0 .
$$

We have achieved (A.17), and hence (A.18), with

$$
\varphi(\sigma)=\frac{a}{2 \sqrt{\sigma}} I_{1}(a \sqrt{\sigma})+\delta_{0}
$$

That is to say, with $L$ as in (A.6),

$$
(\cos t \sqrt{-L}) f=(\cos t \sqrt{-\Delta}) f+a t \int_{0}^{t} \frac{I_{1}\left(a \sqrt{t^{2}-s^{2}}\right)}{\sqrt{t^{2}-s^{2}}}(\cos s \sqrt{-\Delta}) f d s .
$$

In light of this we see that the positivity assertion (A.8) is a consequence of

$$
n=1, f \geq 0 \Longrightarrow \cos t \sqrt{-\Delta} f \geq 0,
$$

which holds because

$$
n=1 \Longrightarrow \cos t \sqrt{-\Delta} f(x)=\frac{f(x+t)+f(x-t)}{2} .
$$

At this point, (A.2) is proven.

Remark. For another approach to formulas such as (A.29), see [1], Vol. 2, pp. 692695.

We now relate the formula (A.29) to the results of Proposition 2.1. We start with the observation that in deriving the identities (A.11) A.29), we made no use of the specific fact that $\Delta$ is the Laplace operator; it could be any selfadjoint operator on a Hilbert space with spectrum bounded from above. In other words, we could take $L_{0}$ to be any selfadjoint operator with spectrum bounded from above and make the replacement

$$
\Delta \mapsto L_{0}, \quad L \mapsto L_{1}=L_{0}+a^{2} .
$$

In this setting, (A.29) becomes (2.9). As for (2.10), it follows from (2.9) by analytic continuation. Alternatively (in fact, equivalently) we can replace $+a^{2}$ by $-a^{2}$ in formulas (A.6), (A.9), A.13), (A.15), and (A.17), then replace (A.25) by (A.22) to get the relevant formula for $\varphi(\sigma)$, in this altered version of (A.17):

$$
\varphi(\sigma)=-\frac{a}{2 \sqrt{\sigma}} J_{1}(a \sqrt{\sigma})+\delta_{0} .
$$

This also gives (2.10).

\section{Appendix B. Local Hardy space, Bmo, and Bmo-Sobolev spaces}

For use in Section 3 we define bmo and bmo-Sobolev spaces here (and relate them to the local Hardy space $\mathfrak{h}^{1}$ ), and present results that yield the interpolation estimates given in (3.12)-(3.15). We present the results in the setting of $\operatorname{bmo}(M)$ and $\mathfrak{h}^{1}(M)$ when $M$ is a Riemannian manifold with bounded geometry, which is a more general setting than required for 43 , but is of potential use in other settings. Proofs of results stated here can be found in the paper 32 . 
The spaces $\mathfrak{h}^{1}\left(\mathbb{R}^{n}\right)$ and bmo $\left(\mathbb{R}^{n}\right)$ were introduced in [14, as variants of the spaces $H^{1}\left(\mathbb{R}^{n}\right)$ and $\operatorname{BMO}\left(\mathbb{R}^{n}\right)$ treated in [12]. One advantage of the spaces $\mathfrak{h}^{1}$ and bmo is that they are invariant under multiplication by bounded Lipschitz functions.

We mention that 15 introduced a variant of $H^{1}$ and BMO for functions on rankone symmetric spaces of noncompact type, though the definition there differs from the Euclidean case in ways that make it problematic to give a unified treatment. (This is perhaps another advantage of using the local spaces.) On the other hand, the work of 15 is directly applicable to the setting of Section 3 .

To define $\mathfrak{h}^{1}(M)$, we set up the following maximal function. Given $f \in L_{\mathrm{loc}}^{1}(M)$, let

$$
\mathcal{G}^{b} f(x)=\sup _{0<r \leq 1} \mathcal{G}_{r} f(x)
$$

where

$$
\mathcal{G}_{r} f(x)=\sup \left\{\left|\int \varphi(y) f(y) d V(y)\right|: \varphi \in \mathcal{F}\left(B_{r}(x)\right)\right\}
$$

with

$$
\mathcal{F}\left(B_{r}(x)\right)=\left\{\varphi \in C_{0}^{1}\left(B_{r}(x)\right):\|\varphi\|_{\text {Lip }} \leq \frac{1}{r^{n+1}}\right\}
$$

We then set

$$
\mathfrak{h}^{1}(M)=\left\{f \in L_{\mathrm{loc}}^{1}(M): \mathcal{G}^{b} f \in L^{1}(M)\right\},
$$

with norm

$$
\|f\|_{\mathfrak{h}^{1}}=\left\|\mathcal{G}^{b} f\right\|_{L^{1}} .
$$

One could replace $C_{0}^{1}\left(B_{r}(x)\right)$ by $\left\{\varphi \in \operatorname{Lip}(M): \operatorname{supp} \varphi \subset B_{r}(x)\right\}$ and get the same result. When $M=\mathbb{R}^{n}$, the space $\mathfrak{h}^{1}(M)$ defined above coincides with the space $\mathfrak{h}^{1}\left(\mathbb{R}^{n}\right)$ defined in $[14$.

To define $\operatorname{bmo}(M)$, we set up the following maximal functions. Given $f \in$ $L_{\text {loc }}^{1}(M)$, let

$$
f^{\#}(x)=\sup _{B \in \mathcal{B}(x)} \frac{1}{V(B)} \int_{B}\left|f-f_{B}\right| d V, \quad f_{B}=\frac{1}{V(B)} \int_{B} f d V,
$$

where

$$
\mathcal{B}(x)=\left\{B_{r}(x): 0<r \leq 1\right\} .
$$

Then define

$$
\mathcal{N} f(x)=f^{\#}(x)+\mathcal{N}_{0} f(x), \quad \mathcal{N}_{0} f(x)=\frac{1}{V\left(B_{1}(x)\right)} \int_{B_{1}(x)}|f| d V .
$$

We set

$$
\operatorname{bmo}(M)=\left\{f \in L_{\text {loc }}^{1}(M): \mathcal{N} f \in L^{\infty}(M)\right\}
$$

with norm

$$
\|f\|_{\text {bmo }}=\|\mathcal{N} f\|_{L^{\infty}} .
$$

In case $M=\mathbb{R}^{n}$, the definition of $\operatorname{bmo}(M)$ given here is equivalent to that of $\operatorname{bmo}\left(\mathbb{R}^{n}\right)$ given in [14]. 
It is useful to make note of some equivalent norms. For example, in place of $f^{\#}$, consider

$$
f^{s}(x)=\sup _{B \in \mathcal{B}(x)} \inf _{c_{B} \in \mathbb{C}} \frac{1}{V(B)} \int_{B}\left|f-c_{B}\right| d V .
$$

Given $B \in \mathcal{B}(x)$ and taking $c_{B}$ to realize this infimum, we have

$$
\left|f_{B}-c_{B}\right|=\left|\frac{1}{V(B)} \int_{B}\left(f-c_{B}\right) d V\right| \leq f^{s}(x),
$$

which gives

$$
f^{s}(x) \leq f^{\#}(x) \leq 2 f^{s}(x) .
$$

It is also useful to note that one can fix $a, b, c \in(0, \infty)$, with $a<b$, and replace $\mathcal{B}(x)$ by

$$
\widetilde{\mathcal{B}}(x)=\left\{Q_{r}^{\alpha}(x): 0<r \leq 1, \alpha \in \mathcal{A}\right\},
$$

where $Q_{r}^{\alpha}(x)$ is a family of measurable sets with the property that for each $r \in(0,1]$,

$$
\begin{aligned}
V\left(Q_{r}^{\alpha}(x)\right) \geq c V\left(B_{r}(x)\right), & Q_{r}^{\alpha}(x) \subset B_{b r}(x), \quad \text { for all } \alpha, \text { and } \\
& B_{a r}(x) \subset Q_{r}^{\alpha}(x), \quad \text { for some } \alpha .
\end{aligned}
$$

One gets functions comparable in size in (B.11) and hence also in (B.6). In connection with this, we recall that the original treatments in [18] and [12] used cubes containing $x$ in place of balls centered at $x$. One consequence of this observation is that the John-Nirenberg estimate, proven in 18 for functions defined on a cube in $\mathbb{R}^{n}$, is applicable in our current situation. We have, for each ball $B \subset M$ of radius $\leq 1$,

$$
\frac{1}{V(B)} \int_{B} e^{\alpha\left|f-f_{B}\right|} d V \leq \gamma,
$$

with

$$
\alpha=\frac{\beta}{\|f\|_{\mathrm{bmo}}}, \quad \beta, \gamma \text { constants. }
$$

Cf. $\left(3^{\prime}\right)$ of 18 .

It is convenient to know that $\mathfrak{h}^{1}(M)$ and $\operatorname{bmo}(M)$ are modules over $\operatorname{Lip}(M) \cap$ $L^{\infty}(M)$. In fact, a more precise result holds. Let $\sigma$ be a modulus of continuity, and say

$$
a \in C^{\sigma}(M) \Longleftrightarrow|a(x)-a(y)| \leq L \sigma(d(x, y)), \text { for } d(x, y) \leq 1,
$$

for some $L \in[0, \infty)$. Define $\|a\|_{C^{\sigma}}$ to be the smallest $L$ for which (B.18) holds (this is a seminorm). We then have the following result, proven in 32 .

Proposition B.1. Let $\sigma$ be a modulus of continuity satisfying the Dini condition

$$
D(\sigma)=\int_{0}^{1} \frac{\sigma(r)}{r} d r<\infty .
$$

We also assume that $\sigma(r) / r$ is monotonically decreasing on $(0,1]$ (or constant). Then

$$
a \in L^{\infty}(M) \cap C^{\sigma}(M), f \in \mathfrak{h}^{1}(M) \Longrightarrow a f \in \mathfrak{h}^{1}(M) .
$$


On the other hand, if $a \in L^{\infty}(M) \cap C^{\sigma}(M)$ with

$$
\sigma(r)=\left(\log \frac{1}{r}\right)^{-1}, \quad 0<r \leq \frac{1}{2},
$$

then

$$
f \in \operatorname{bmo}(M) \Longrightarrow a f \in \operatorname{bmo}(M) .
$$

The proof of (B.20) is fairly straightforward; Schur's lemma is involved. The proof of (B.22) uses the John-Nirenberg estimate (B.16).

Remark. Note that the Dini condition (B.19) just barely fails for $\sigma(r)$ given by (B.21). $\mathfrak{h}^{1}$-bmo duality, discussed below, allows one to amalgamate these results.

Using Proposition B.1, we can establish the following result. To state it, we define a tame partition of unity on a manifold $M$ with bounded geometry to be a partition of unity whose elements, pulled back by exponential maps, are bounded in $C_{0}^{\infty}$ of a ball in $\mathbb{R}^{n}$ and whose supports have a bounded number of overlaps.

Proposition B.2. Let $\left\{\varphi_{k}: k \in \mathbb{Z}^{+}\right\}$be a tame partition of unity. Given $f \in$ $L_{\text {loc }}^{1}(M)$, we have

$$
f \in \mathfrak{h}^{1}(M) \Longleftrightarrow \sum_{k}\left\|\varphi_{k} f\right\|_{\mathfrak{h}^{1}}<\infty
$$

and

$$
\|f\|_{\mathfrak{h}^{1}} \approx \sum_{k}\left\|\varphi_{k} f\right\|_{\mathfrak{h}^{1}}
$$

Furthermore,

$$
f \in \operatorname{bmo}(M) \Longleftrightarrow \sup _{k}\left\|\varphi_{k} f\right\|_{\text {bmo }}<\infty
$$

and

$$
\|f\|_{\mathrm{bmo}} \approx \sup _{k}\left\|\varphi_{k} f\right\|_{\mathrm{bmo}} .
$$

Proposition B.2 combines nicely with the following elementary result. In the statement, we use an isometric isomorphism of the $n$-dimensional inner product space $T_{p} M$ with $\mathbb{R}^{n}$, determined uniquely up to the action of $O(n)$.

Proposition B.3. We have, uniformly in $k \in \mathbb{Z}^{+}$,

$$
\left\|\varphi_{k} f\right\|_{\mathfrak{h}^{1}(M)} \approx\left\|\left(\varphi_{k} f\right) \circ \operatorname{Exp}_{p_{k}}\right\|_{\mathfrak{h}^{1}\left(\mathbb{R}^{n}\right)}
$$

and

$$
\left\|\varphi_{k} f\right\|_{\mathrm{bmo}(M)} \approx\left\|\left(\varphi_{k} f\right) \circ \operatorname{Exp}_{p_{k}}\right\|_{\mathrm{bmo}\left(\mathbb{R}^{n}\right)} .
$$

Corollary B.4. In the setting of Proposition B.2,

$$
\|f\|_{\mathfrak{h}^{1}(M)} \approx \sum_{k}\left\|\left(\varphi_{k} f\right) \circ \operatorname{Exp}_{p_{k}}\right\|_{\mathfrak{h}^{1}\left(\mathbb{R}^{n}\right)}
$$

and

$$
\|f\|_{\mathrm{bmo}(M)} \approx \sup _{k}\left\|\left(\varphi_{k} f\right) \circ \operatorname{Exp}_{p_{k}}\right\|_{\mathrm{bmo}\left(\mathbb{R}^{n}\right)} .
$$


These results open the door to making use of Euclidean results of [14, and, by extension, results of [12. For example, we can prove the duality

$$
\mathfrak{h}^{1}(M)^{\prime}=\operatorname{bmo}(M),
$$

using Corollary B.4 and the result of [14 that (B.31) holds for $M=\mathbb{R}^{n}$, itself a consequence of the famous result

$$
H^{1}\left(\mathbb{R}^{n}\right)^{\prime}=\operatorname{BMO}\left(\mathbb{R}^{n}\right)
$$

of [12]. Furthermore, the result of [14] that

$$
\begin{aligned}
P \in O P S_{1,0}^{0}\left(\mathbb{R}^{n}\right) \Longrightarrow & P: \mathfrak{h}^{1}\left(\mathbb{R}^{n}\right) \rightarrow \mathfrak{h}^{1}\left(\mathbb{R}^{n}\right) \text { and } \\
& P: \operatorname{bmo}\left(\mathbb{R}^{n}\right) \rightarrow \operatorname{bmo}\left(\mathbb{R}^{n}\right)
\end{aligned}
$$

can be used to prove important results on the action on $\mathfrak{h}^{1}(M)$ and $\operatorname{bmo}(M)$ of certain classes of pseudodifferential operators, which we now define.

Given an operator $P: C_{0}^{\infty}(M) \rightarrow \mathcal{D}^{\prime}(M)$, we say $P \in \Psi_{\#}^{m}(M)$ provided the following conditions hold. First we assume its Schwartz kernel $K_{P} \in \mathcal{D}^{\prime}(M \times M)$ satisfies

$$
\begin{gathered}
\operatorname{supp} K_{P} \subset\{(x, y) \in M \times M: d(x, y) \leq 1\}, \\
\operatorname{sing} \operatorname{supp} K_{P} \subset \operatorname{diag}(M \times M)=\{(x, x): x \in M\} .
\end{gathered}
$$

Next, we assume that, for each $p \in M$,

$$
M_{\varphi_{1}} P M_{\varphi_{2}} \in O P S_{1,0}^{m}\left(\mathbb{R}^{n}\right),
$$

with uniform bounds, independent of $p \in M$, where this statement has the following meaning.

A Riemannian manifold with bounded geometry is covered by balls of radius $R_{0}>0$ such that the Riemannian metric tensor, pulled back by the exponential map to a metric tensor on $B_{R_{0}} \subset T_{p} M \approx \mathbb{R}^{n}$, is bounded in $C^{\infty}\left(B_{R_{0}}\right)$, with bounded inverse, independent of $p$. Scaling, we take $R_{0} \geq 4$. Thus functions supported on $B_{4}(p) \subset M$ are identified with functions supported on $B_{4}(0) \subset \mathbb{R}^{n}$. We pick $\varphi_{j} \in C_{0}^{\infty}\left(B_{4}(0)\right) \approx C_{0}^{\infty}\left(B_{4}(p)\right)$, equal to 1 on $B_{2}(p)$, and set $M_{\varphi_{j}} f=\varphi_{j} f$, and use these identifications to regard $M_{\varphi_{1}} P M_{\varphi_{2}}$ as operating on functions on $\mathbb{R}^{n}$.

Then, given $W \geq 0$, set

$$
\Psi_{W}^{m}(M)=\left\{P^{\#}+P^{b}: P^{\#} \in \Psi_{\#}^{m}(M), P^{b} \in \Psi_{W}^{-\infty}(M)\right\},
$$

where we say $P^{b} \in \Psi_{W}^{-\infty}(M)$ provided it has the form

$$
P^{b} f(x)=\int_{M} k^{b}(x, y) f(y) d V(y),
$$

where $k^{b} \in C^{\infty}(M \times M)$ satisfies

$$
\left|k^{b}(x, y)\right| \leq C_{j}\langle d(x, y)\rangle^{-j} e^{-W d(x, y)},
$$

and also such estimates for all $x$ and $y$-derivatives of $k^{b}(x, y)$ (say in local exponential coordinates). The need for the exponential decrease follows from the fact that for a manifold with bounded geometry, there is a volume estimate of the form

$$
\operatorname{Vol}\left(B_{r}(y)\right) \leq C\langle r\rangle^{\mu} e^{\kappa r},
$$

and if $\kappa>0$ one needs such decay for good operator properties. We mention that

$$
M=\mathcal{H}_{n} \Longrightarrow \kappa=n-1 \text {. }
$$


Here are useful operator boundedness results from [32.

Proposition B.5. Take $\kappa$ as in (B.39). Then

$$
\begin{array}{r}
P \in \Psi_{W}^{0}(M), W \geq \kappa \Longrightarrow \\
P: \mathfrak{h}^{1}(M) \rightarrow \mathfrak{h}^{1}(M) \text { and } \\
\\
P: \operatorname{bmo}(M) \rightarrow \operatorname{bmo}(M) .
\end{array}
$$

As shown in 32 ,

$$
\lambda>W^{2} \Longrightarrow(\lambda I-\Delta)^{-s / 2} \in \Psi_{W}^{-s}(M) .
$$

We can define Hardy and bmo-Sobolev spaces. We can take $\lambda>\kappa^{2}$ and set

$$
\begin{aligned}
\mathfrak{h}^{s, 1}(M) & =(\lambda I-\Delta)^{-s / 2} \mathfrak{h}^{1}(M), \\
\mathfrak{h}^{s, \infty}(M) & =(\lambda I-\Delta)^{-s / 2} \operatorname{bmo}(M) .
\end{aligned}
$$

These spaces are shown to be independent of the choice of $\lambda$, as long as $\lambda>\kappa^{2}$. One can show that, for $k \in \mathbb{Z}^{+}$,

$$
\begin{aligned}
\mathfrak{h}^{k, 1}(M) & =\left\{u \in \mathfrak{h}^{1}(M): \mathcal{L} u \in \mathfrak{h}^{1}(M), \forall \mathcal{L} \in \mathcal{V}^{k}(M)\right\}, \\
\mathfrak{h}^{h, \infty}(M) & =\left\{u \in \operatorname{bmo}(M): \mathcal{L} u \in \operatorname{bmo}(M), \forall \mathcal{L} \in \mathcal{V}^{k}(M)\right\}
\end{aligned}
$$

Extending Proposition B.5, we have:

Proposition B.6. Take $m, s \in \mathbb{R}$ and assume $W \geq \kappa$. Then

$$
\begin{aligned}
P \in \Psi_{W}^{m}(M) \Longrightarrow & P: \mathfrak{h}^{s, 1}(M) \rightarrow \mathfrak{h}^{s-m, 1}(M) \text { and } \\
& P: \mathfrak{h}^{s, \infty}(M) \rightarrow \mathfrak{h}^{s-m, \infty}(M) .
\end{aligned}
$$

Another result of [32] is the following analogue of the sharp maximal function estimate in $L^{p}$ of [12]. (For $M=\mathcal{H}^{n}$, this also follows from results of [15].)

Proposition B.7. Assume $p \in(1, \infty), f \in L_{l o c}^{1}(M)$, and $\mathcal{N} f \in L^{p}(M)$. Then $f \in L^{p}(M)$ and

$$
\|f\|_{L^{p}(M)} \leq C_{p}\|\mathcal{N} f\|_{L^{p}(M)} .
$$

Using this, 32] establishes the following interpolation result, a variant of Corollary 2 of [12]:

Proposition B.8. Take $s \in \mathbb{R}$. Assume we have a bounded operator

$$
R: L^{2}(M) \rightarrow L^{2}(M), \quad R: L^{1}(M) \rightarrow \mathfrak{h}^{s, \infty}(M),
$$

satisfying

$$
\|R f\|_{L^{2}} \leq M_{1}\|f\|_{L^{2}}, \quad\|R f\|_{\mathfrak{h}^{s, \infty}} \leq M_{0}\|f\|_{L^{1}} .
$$

Then, for $\theta \in(0,1)$,

$$
R: L^{p(\theta)}(M) \rightarrow H^{(1-\theta) s, p(\theta)^{\prime}}(M), \quad p(\theta)=\frac{2}{2-\theta}, \quad p(\theta)^{\prime}=\frac{2}{\theta},
$$

and (with $C_{\theta} \in(0, \infty)$ independent of $R$ and $f$ ),

$$
\|R f\|_{H^{(1-\theta) s, p(\theta)^{\prime}}} \leq C_{\theta} M_{1}^{\theta} M_{0}^{1-\theta}\|f\|_{L^{p(\theta)}} .
$$

It is this result which is used in (3.12)-(3.15).

Remark. The results mentioned above are counterparts to $L^{p}$-Sobolev space results, which are also covered in 32, as well as in other places. There are sharper results covered in [32, which we will not discuss in detail here. We mention that (3.48) is given as Proposition C.1 of [32]. 


\section{Appendix C. Generalities on global smooth solutions}

For use in 88 , we present some general results on when one can guarantee that solutions to a nonlinear wave equation for $t \in[0, T)$ do not break down at $t=T$. We consider solutions of semilinear wave equations of the form

$$
\left(\partial_{t}^{2}-\Delta\right) u=F(u), \quad u(0)=f, \quad \partial_{t} u(0)=g,
$$

on $\mathbb{R} \times M$, where $M$ is a complete Riemannian manifold, of dimension $n$, with bounded geometry and $\Delta$ is its Laplace-Beltrami operator. We assume that $F$ is smooth and $F(0)=0$. We take $f, g \in C_{0}^{\infty}(M)$. In this setting, local solvability of (C.1) is well known.

We assume the following special property on $M$ :

$$
\operatorname{Spec}(-\Delta) \subset[A, \infty), \quad A>0 .
$$

Recall that our application of the material in this appendix will be to the case $M=\mathcal{H}^{3}$. In case $M=\mathcal{H}^{n}$, an $n$-dimensional hyperbolic space having constant sectional curvature -1 , then equality holds in (C.2), with $A=(n-1)^{2} / 4$ for $n=3, A=1$. (Cf. 30], Chapter 8, §5.)

To start, we assume that $u$ is a smooth solution to C.1 on $[0, T) \times M$, with compact support in $M$ for each $t$ (by finite propagation speed) and examine some energy estimates. First,

$$
\begin{aligned}
\frac{d}{d t}\left(\left\|\partial_{t} u(t)\right\|_{L^{2}}^{2}+\|\nabla u(t)\|_{L^{2}}^{2}\right) & =2\left(u_{t t}-\Delta u, u_{t}\right)_{L^{2}} \\
& =2\left(F(u), u_{t}\right)_{L^{2}} \\
& \leq 2\|F(u(t))\|_{L^{2}}\left\|\partial_{t} u(t)\right\|_{L^{2}}
\end{aligned}
$$

Next,

$$
\begin{aligned}
\frac{d}{d t}\left(\|\Delta u\|_{L^{2}}^{2}+\left\|\nabla u_{t}\right\|_{L^{2}}^{2}\right) & =2\left(\Delta u_{t}, \Delta u\right)+2\left(\nabla u_{t t}, \nabla u_{t}\right) \\
& =2\left(\Delta u_{t}, \Delta u-u_{t t}\right) \\
& =-2\left(F(u), \Delta u_{t}\right) \\
& =2\left(\nabla F(u), \nabla u_{t}\right) \\
& =2\left(F^{\prime}(u) \nabla u, \nabla u_{t}\right) \\
& \leq 2\left\|F^{\prime}(u(t))\right\|_{L^{4}}\|\nabla u(t)\|_{L^{4}}\left\|\nabla u_{t}\right\|_{L^{2}} .
\end{aligned}
$$

We bring in the following well-known general result. (Cf. [30], Chapter 16, §3.)

Proposition C.1. Smooth solutions to (C.1) on $[0, T)$ do not break down as $t \rightarrow T$ as long as $\|u(t)\|_{L^{\infty}}$ is bounded.

Since $\mathcal{D}(\Delta) \subset L^{\infty}(M)$ for $n \leq 3$, we have:

Corollary C.2. Assume $n \leq 3$. Then smooth solutions to (C.1) on $[0, T)$ do not break down as $t \rightarrow T$, as long as there exist $K_{j}<\infty$ such that

$$
\|F(u(t))\|_{L^{2}} \leq K_{1}, \quad\left\|F^{\prime}(u(t))\right\|_{L^{4}} \leq K_{2}, \quad\|\nabla u(t)\|_{L^{4}} \leq K_{3} .
$$

In more detail, set

$$
\begin{aligned}
& E_{1}(t)=\left\|\partial_{t} u(t)\right\|_{L^{2}}^{2}+\|\nabla u(t)\|_{L^{2}}^{2}, \\
& E_{2}(t)=\|\Delta u(t)\|_{L^{2}}^{2}+\left\|\nabla u_{t}(t)\right\|_{L^{2}}^{2}
\end{aligned}
$$


Then we have

$$
\begin{aligned}
\frac{d}{d t} E_{1}(t) & \leq 2 K_{1} \sqrt{E_{1}(t)}, \\
\frac{d}{d t} E_{2}(t) & \leq 2 K_{2} K_{3} \sqrt{E_{2}(t)},
\end{aligned}
$$

and Gronwall's inequality gives

$$
\begin{aligned}
& E_{1}(t) \leq\left(K_{1} t+E_{1}(0)^{1 / 2}\right)^{2}, \\
& E_{2}(t) \leq\left(K_{2} K_{3} t+E_{2}(0)^{1 / 2}\right)^{2} .
\end{aligned}
$$

Corollary $\mathrm{C.2}$ is applied in $₫ 8$, in the form of Proposition 8.1

\section{REFERENCES}

[1] J.-P. Anker and V. Pierfelice, Nonlinear Schrödinger equation on real hyperbolic spaces, Ann. Inst. H. Poincaré Anal. Non Linéaire 26 (2009), 1853-1869. MR2566713

[2] V. Banica, The nonlinear Schrödinger equation on hyperbolic space, Comm. Partial Differential Equations 32 (2007), 1643-1677. MR 2372482 (2008m:35323)

[3] V. Banica, R. Carles, and T. Duyckaerts, On scattering for NLS: from Euclidean to hyperbolic space, Discrete Contin. Dyn. Syst. 24 (2009), 1113-1127. MR2505694

[4] V. Banica, R. Carles, and G. Staffilani, Scattering theory for radial nonlinear Schrödinger equations on hyperbolic space, Geom. Funct. Anal. 18 (2008), 367-399. MR2421543

[5] V. Banica and T. Duyckaerts, Weighted Strichartz estimates for radial Schrödinger equation on noncompact manifolds, Dyn. Partial Differ. Equ. 4 (2007), 335-359. MR.2376801 (2008j:58036)

[6] A. Bobrowski, Functional Analysis for Probability and Stochastic Processes, Cambridge Univ. Press, 2005. MR2176612 (2006g:60001)

[7] J. Cheeger, M. Gromov, and M. Taylor, Finite propagation speed, kernel estimates for functions of the Laplace operator, and the geometry of complete Riemannian manifolds, J. Diff. Geom. 17 (1982), 15-53. MR.658471 (84b:58109)

[8] M. Christ and A. Kiselev, Maximal operators associated to filtrations, J. Funct. Anal. 179 (2001), 409-425. MR:1809116 (2001i:47054)

[9] M. Christ and M. Weinstein, Dispersion of small amplitude solutions of the generalized Korteweg-de Vries equations, J. Funct. Anal. 100 (1991), 87-109. MR.1124294 (92h:35203)

[10] R. Coifman and G. Weiss, Extensions of Hardy spaces and their use in analysis, Bull. Amer. Math. Soc. 83 (1977), 569-645. MR0447954(56:6264)

[11] R. Courant and D. Hilbert, Methods of Mathematical Physics, Vols. 1-2, J. Wiley, New York, 1966. MR0195654 (33:3852)

[12] C. Fefferman and E. Stein, $H^{p}$ spaces of several variables, Acta Math. 129 (1972), 137-193. MR0447953 (56:6263)

[13] V. Georgiev, H. Lindblad, and C. D. Sogge, Weighted Strichartz estimates and global existence for semilinear wave equations, Amer. J. Math. 119 (1997), 1291-1319. MR 1481816 (99f:35134)

[14] D. Goldberg, A local version of real Hardy spaces, Duke Math. J. 46 (1979), 27-42. MR.523600 (80h:46052)

[15] A. Ionescu, Fourier integral operators on noncompact symmetric spaces of real rank one, J. Funct. Anal. 174 (2000), 274-300. MR.1767376 (2001h:43009)

[16] A. Ionescu and G. Staffilani, Semilinear Schrödinger flows on hyperbolic space: Scattering in $H^{1}$, Math. Ann. 345 (2009), 133-158. MR2520054 (2010c:35031)

[17] F. John, Blow-up of solutions of nonlinear wave equations in three space dimensions, Manuscripta Math. 28 (1979), 235-265. MR.535704 (80i:35114)

[18] F. John and L. Nirenberg, On functions of bounded mean oscillation, Comm. Pure Appl. Math. 24 (1961), 415-426. MR0131498 (24:A1348)

[19] M. Keel and T. Tao, Endpoint Strichartz estimates, Amer. J. Math. 120 (1998), 955-980. MR.1646048(2000d:35018)

[20] M. Pinsky and M. Taylor, Pointwise Fourier inversion: a wave equation approach, J. Fourier Anal. 3 (1997), 647-703. MR 1481629 (99d:42019) 
[21] G. Ponce and T. Sideris, Local regularity of nonlinear wave equations in three space dimensions, Comm. PDE 18 (1993), 169-177. MR.1211729 (95a:35092)

[22] H. Smith and C. Sogge, Global Strichartz estimates for nontrapping perturbations of the Laplacian, Comm. PDE 25 (2000), 2127-2183. MR1789924 (2001j:35180)

[23] C. Sogge, Lectures on Nonlinear Wave Equations, International Press, Boston, 1995. Second edition, 2008. MR1715192(2000g:35153)

[24] G. Staffilani, On the generalized Korteweg-de Vries-type equations, Differential Integral Equations 10 (1997), 777-796. MR1741772 (2001a:35005)

[25] R. Strichartz, Restrictions of Fourier transforms to quadratic surfaces and decay of solutions of wave equations, Duke Math. J. 44 (1977), 705-714. MR0512086 (58:23577)

[26] R. Strichartz, Analysis of the Laplacian on the complete Riemannian manifold, J. Funct. Anal. 52 (1983), 48-79. MR705991 (84m:58138)

[27] D. Tataru, Strichartz estimates in the hyperbolic space and global existence for the semilinear wave equation, Trans. Amer. Math. Soc. 353 (2001), 795-807. MR.1804518 (2001k:35218)

[28] M. Taylor, $L^{p}$ estimates on functions of the Laplace operator, Duke Math. J. 58 (1989), 773-793. MR1016445(91d:58253)

[29] M. Taylor, Pseudodifferential Operators and Nonlinear PDE, Birkhäuser, Boston, 1991. MR1121019 (92j:35193)

[30] M. Taylor, Partial Differential Equations, Vols. 1-3, Springer-Verlag, New York, 1996. MR:1395147(98b:35002a)

[31] M. Taylor, Tools for PDE, Math. Surv. Monogr. \#81, American Mathematical Society, Providence, RI, 2000. MR.1766415 (2001g:35004)

[32] M. Taylor, Hardy spaces and bmo on manifolds with bounded geometry, J. Geometric Anal. 19 (2009), 137-190. MR2465300(2009k:58053)

Department of Mathematics, University of North Carolina, Chapel Hill, North CarOLINA 27599-3250

E-mail address: metcalfe@email.unc.edu

Department of Mathematics, University of North Carolina, Chapel Hill, North CarOLINA 27599-3250

E-mail address: met@email.unc.edu 